San Jose State University

SJSU ScholarWorks

Master's Theses

Master's Theses and Graduate Research

1992

\title{
The effects of rest interval length on acute physiological responses to heavy-resistance exercise
}

Hugo F. Quevedo

San Jose State University

Follow this and additional works at: https://scholarworks.sjsu.edu/etd_theses

\section{Recommended Citation}

Quevedo, Hugo F., "The effects of rest interval length on acute physiological responses to heavyresistance exercise" (1992). Master's Theses. 353.

DOI: https://doi.org/10.31979/etd.a6hw-4jm4

https://scholarworks.sjsu.edu/etd_theses/353

This Thesis is brought to you for free and open access by the Master's Theses and Graduate Research at SJSU ScholarWorks. It has been accepted for inclusion in Master's Theses by an authorized administrator of SJSU ScholarWorks. For more information, please contact scholarworks@sjsu.edu. 


\section{INFORMATION TO USERS}

This manuscript has been reproduced from the microfilm master. UMI films the text directly from the original or copy submitted. Thus, some thesis and dissertation copies are in typewriter face, while others may be from any type of computer printer.

The quality of this reproduction is dependent upon the quality of the copy submitted. Broken or indistinct print, colored or poor quality illustrations and photographs, print bleedthrough, substandard margins, and improper alignment can adversely affect reproduction.

In the unlikely event that the author did not send UMI a complete manuscript and there are missing pages, these will be noted. Also, if unauthorized copyright material had to be removed, a note will indicate the deletion.

Oversize materiais (e.g., maps, drawings, charts) are reproduced by sectioning the original, beginning at the upper left-hand corner and continuing from left to right in equal sections with small overlaps. Each original is also photographed in one exposure and is included in reduced form at the back of the book.

Photographs included in the original manuscript have been reproduced xerographically in this copy. Higher quality,.6" $\times$ 9" black and white photographic prints are available for any photographs or illustrations appearing in this copy for an additional charge. Contact UMI directly to order.

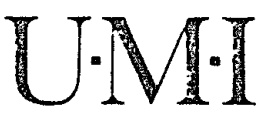

University Microfilms International

- A Bell \& Howell Information Company

300 North Zeeb Road. Ann Arbor. MI 48106-1346 USA

$313 / 761-4700 \quad 800 / 521-0600$ 

Order Number 1348714

The effects of rest interval length on acute physiological responses to heavy-resistance exercise

Quevedo, Hugo Francisco, M.A.

San Jose State University, 1992

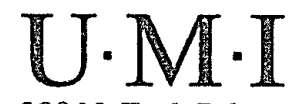

300 N. Zeeb Rd.

Ann Arbor, MI 48106 



\title{
THE EFFECTS OF REST INTERVAL LENGTH ON ACUTE PHYSIOLOGICAL RESPONSES TO HEAVY-RESISTANCE EXERCISE
}

\author{
A Thesis \\ Presented to \\ The Faculty of the Department of Human Performance \\ San Jose State University
}

\author{
In Partial Fulfillment \\ of the Requirements for the Degree \\ Master of Arts
}

By

Hugo F. Quevedo

May, 1992 
APPROVED FOR THE DEPARTMENT OF HUMAN PERFORMANCE
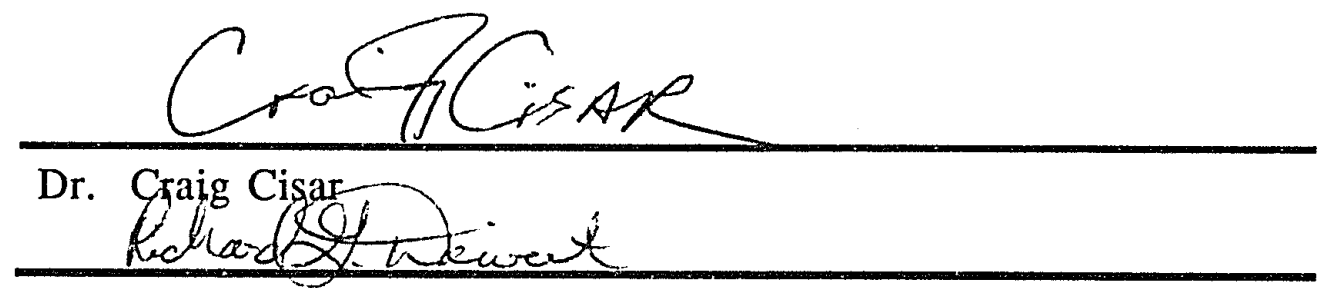

Dr. Richard Deivert Pcierer G. SNu

Dr. Phillip Sienna

APPROVED BY THE UNIVERSITY

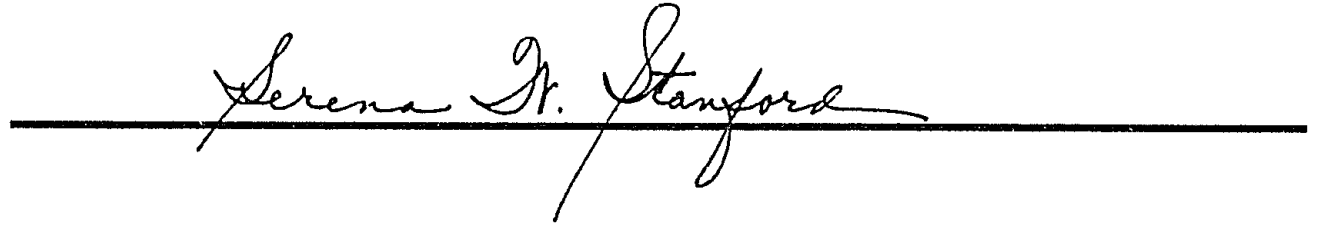




\section{ABSTRACT \\ THE EFFECTS OF REST INTERVAL LENGTH ON ACUTE PHYSIOLOGICAL RESPONSES TO HEAVY-RESISTANCE EXERCISE}

by Hugo F. Quevedo

The physiological effects of resistance training protocols using 1-min (TP1) and 3-min (TP3) rest interval lengths were examined in 11 male weight lifters aged 20 to $35 \mathrm{yr}$. Both protocols consisted of three sets of 10 exercises using a 10-repetition maximum load. Blood lactate (BLA), heart rate (HR), systolic blood pressure (SBP), cardiac cost (CC), and rating of perceived exertion (RPE) were measured immediately at the completion of the third set of exercises at stations two, four, six, eight, and ten. The physiological variables were also measured at rest and after 5-min of post-exercise recovery. Two-way analyses of variance revealed that BLA, HR, SBP, $\mathrm{CC}$, and RPE increased significantly $(\underline{p}<.05)$ during both training protocols. In addition, BLA, HR, CC, and RPE were significantly higher during TP1 compared to TP3. The higher cardiovascular and metabolic demands during TP1 indicate that this protocol may be more appropriate for improving anaerobic capacity. 
This thesis is dedicated to my family and to the people striving to achieve human excellence. 
Table of Content

\section{CHAPTER}

Page

I. Introduction..............................................................................

Background for the Study.................................... 1

Significance of the Study..................................... 4

Statement of the Problem................................... 7

Approach to the Problem...................................... 7

Statement of the Null Hypotheses................... 8

Delimitations of the Study.................................. 8

Limitations of the Study..................................... 9

Assumptions of the Study.................................. 9

Definition of terms................................................. 9

II. Review of Literature.......................................................... 14

Introduction............................................................. 14

Background of Energy Metabolism Studies.. 15

Heavy Resistance Exercise and Metabolism. 18

Summary............................................................... 26

III. Methods............................................................................ 31

Introduction ........................................................ 31

Subjects........................................................... 31

Experimental Design............................................... 31

One Repetition Maximum................................... 32 


\section{Chapter}

Training Sessions................................................ 32

Heart Rate Assessment....................................... 33

Blood Lactate Determination.............................. 34

Blood Pressure Assessment.............................. 34

Rate of Perceived Exertion................................. 34

Statistical Analysis.............................................. 35

IV. Analysis of Data.............................................................. 36

Introduction....................................................... 36

Level of Significance.......................................... 36

Analysis of Data..................................................... 36

Summary of Findings........................................ 53

Discussion of Findings....................................... 55

Conclusions........................................................... 59

Weaknesses............................................................ 60

Recommendations for Future Research......... 61

References................................................................................ 62 
Appendices

A. Pre-exercise Testing Health Status Questionnaire........... 68

B. Informed Consent Form........................................................... 75

C. Training Protocol with One Minute Rest Intervals......... 82

D. Training Protocol with Three Minute Rest Intervals.... 84

E. Standardized Warm-Up Protocol.......................................... 86

F. Standardized Cool-Down Protocol.............................................. 88

G. Rate of Perceived Exertion Graph........................................ 90

H. Heart Rate Responses Graph................................................... 92

I. Blood Lactate Concentration Levels Graph......................... 94

J. Systolic Blood Pressure Responses Graph........................... 96

K. Cardiac Cost Responses Graph.................................................. 98

L. Anova Summary Tables.......................................................... 100 
1. Muscle Metabolite Concentration

Before and After Exercise...................................... 20

2. Heart rate (HR) and Lactate (LA) Levels in Five Trained and Five Untrained Men After

Squatting Exercise.................................................... 22

3. Maximal Oxygen Uptake Rate (VO2), Blood

Lactate (LA), and Heart Rate (HR) Responses

During and Following Four Intensities of

Weight Training.

4. Summary of the Results of Acute

Physiological Responses to Heavy Resistance

Exercise..................................................................... 29

5. Physical Characteristics of the Subjects.............. 37

6. Maximal Strength and Mean Training Loads at Each Station................................................ 38

7. Mean Training Protocols Performed by the Subjects................................................................... 39

8. Results of Paired t-tests for Both Training Protocols................................................................. 40 
Tables

Page

9. Responses of Blood Lactate Concentration

During the Training Protocols.............................. 45

10. Heart Rate Responses During the Training Protocols.......................................................................... 46

11. Rating of Perceived Exertion During the Training Protocols................................................... 48

12. Systolic Blood Pressure Responses During the Training Protocols............................................ 50

13. Cardiac Cost During the Training Protocols.... 52 


\section{CHAPTER I}

\section{Introduction}

This chapter is organized in several sections as follows: background for the study, significance of the study, statement of the problem, approach to the problem, statement of the null hypotheses, delimitations of the study, limitations of the study, assumptions of the study, and definition of terms.

\section{Background for the Study}

Exercise prescription in weight training requires a need analysis. First, a biomechanical analysis to single out the muscle or muscle groups that are most involved in the sport or activity to be performed, then a metabolic analysis of the sport or activity to be performed is needed. One of the easiest ways to determine the metabolic profile of the "sport" is to define the duration of the physical activity itself, since the time of performance is related to the energy-yielding systems involved (Dudley \& Murray, 1982; Fox, Bowers, \& Foss, 1988; Jones, McCarney, \& McComas, 1986). The second step in the analysis is to develop the optimal program which meets the needs and demands of the sport. Several factors need to be taken into consideration. At what weight will the exercises be performed and how many sets will be done? What will be the choice and order of the exercises? And finally, how much of a rest interval will be allowed and where they will be inserted in the program. 
Researchers agree that a low repetitions-high intensity workout will be more successful at increasing strength, while high repetitions-low intensity programs will mostly develop endurance (Stone, O' Bryant, Garhammer, McMillan, \& Rozenek, 1982; Tesch, 1988). It is important to note that these two different weight training regimens are not mutually exclusive. That is, muscular strength programs will develop muscular endurance to a lesser degree and muscular endurance programs will also develop muscular strength to a lesser degree (Anderson \& Kearney, 1982). Heavy resistance exercise is characterized by short bouts of intense exercise followed by a rest interval that varies accordingly to the orientation of the training program. Heavy resistance exercises are associated with a high rate of energy utilization through phosphagen breakdown and activation of glycogenolysis (Dudley \& Murray, 1982; Tesch, Colliander, \& Kaiser, 1986). Rest intervais along with the resistance load play a major role in the energy source(s) stressed in a heavy resistance exercise program. For instance, short rest periods and heavier loads place a greater stress on the anaerobic lactic acid system. Conversely, longer rest periods with a similar workload stresses primarily the anaerobic alactic acid system (Kraemer, 1983).

In dynamic and static short-term exercise of high intensity, phosphagen breakdown, accumulation of intermediates in the glycolytic pathway, and excess lactate concentration have been demonstrated. For instance, when performing maximal exercise, the breakdown of phosphagens (ATP-PC) was already maximal after two 
minutes of work and the accumulation of lactate in the muscle and blood increased continuously until exhaustion (Karlsson \& Saltin, 1970). The extent to which these changes occur appears to be dependent upon the relative power output, the work to rest ratio, rate of muscle blood flow, and possibly the degree of muscle tension.

There are few reports on the metabolic demand of heavy resistance exercise. Keul, Haralambie, Bruder, and Gottstein (1978) examined the performance of resistive exercises (bench press, dead lift, and squat) in a pyramid fashion (repetitions of $10,5,3,2,1$, and 1 per set for each exercise). The initial loads where 50, 90, and 60 $\mathrm{kg}$ which increased at 8,10 , and $10 \mathrm{~kg}$, respectively. The rest between sets and exercises was two and five minutes, respectively, and induced only modest increases in blood lactate. The authors suggested that the energy requirements during strength exercises appeared to be met exclusively through the depletion of the energyrich phosphates.

Conversely, when one set of 14 exercises was performed between 8-12 times with short rest intervals (5 seconds) an increase in blood lactate concentration of 16-fold was found (Hurley, Seals, Ehsani, Cartier, Dalski, Hagberg, \& Holloszy, 1984). Similarly, during a whole body exercise protocol consisting of three sets of $10 \mathrm{RM}$ (variable loads) with 10 seconds rest between sets and 30-60 second rest intervals between exercises, plasma lactate concentrations were significantly elevated to as high as $21 \mathrm{mmol} / \mathrm{L}$ (Kraemer, Noble, Clark, \& Culver, 1987). These results indicate that the degree of 
lactate concentration during heavy resistance exercise is heavily dependent on the intensity (workload), exercise time, and exercise to rest ratio of a particular session. Knowing the magnitude of the changes of blood lactate consequent to heavy resistance exercise may produce a better understanding as to whether the anaerobic alactic (ATP-PC) or lactic (Lactic acid) energy system is being stressed and may have important practical implications.

To date, the effects of acute physiological responses to one minute and to a three minute rest interval lengths during a heavyresistance exercise protocol have not been studied. Therefore, the relationship of lactate levels to rest interval length has not yet been clearly established.

\section{Significance of the Study}

There are four general considerations that are important to a!! training programs: (1) the basic principles of training; (2) the various training phases; (3) preliminary activity or warm-up; and (4) cooldown (Fox, Bowers, \& Foss, 1988). In heavy resistance exercise, one of the basic principles of training is the principle of "specificity of training"; that is, the training protocol should mimic as closely as possible the energy source(s) to be stressed in the performance of the actual event. Therefore, the person prescribing the exercise needs to recognize the major energy source utilized to perform a given activity and then, through the overload principle, construct a program that will develop that particular energy source. In 
determining the predominant energy system, regardless of the event, the duration of performance is related to the energy-yielding system(s) involved. Therefore, the energy sources for a given activity are time-dependent.

Unfortunately what seems to be easy in theory is not so in a practical sense. Typically, in a heavy resistance exercise program designed to improve muscular hypertrophy, the protocol includes low resistance-high repetitions ( 8 to 12 ) and short rest periods (less than one minute). However, programs oriented to develop muscular strength include high resistance-low repetitions (less than 8 ) and long rest intervals (more than three minutes) (Anderson \& Kearny, 1982). The energy utilized in these protocols comes predominantly from the anaerobic lactacid and the anaerobic alactacid energy systems, respectively (Kraemer, 1983).

There are some inconsistencies as to the acute physiological responses to heavy resistance exercise. The findings of past research have shown that while, examining a protocol consisting of sets of ten repetitions with 2.5 minutes of standing rest between sets performed to exhaustion, heavy resistance exercise strongly activates glycolysis. Therefore, appropriate heavy resistance exercise may influence anaerobic capacity ( Stone, Pierce, Godsen, Wilson, Blessing, Rozenek, \& Chromiak, 1987). Tesch et al. (1986), while investigating the acute metabolic response to heavy resistance exercise, similarly concluded that high intensity heavy resistance exercise is associated with a high rate of energy utilization through phosphagen 
breakdown and activation of glycogenolysis. Conversely, in a study investigating the effect of weight lifting exercise on heart rate and metabolism in experienced weight lifters, Keul et al. (1978) used a protocol with five minute rest intervals and concluded that LA concentrations in blood are barely changed after several workloads. Further, anaerobic capacity as calculated from maximal blood lactate concentration was not strongly influenced (Keul et al., 1978).

There are few studies available on acute physiological responses, such as heart rate, lactate levels, and rating of perceived exertion (RPE) to heavy resistance exercise (Guezennec, Leger, Lhoste, Aymonod, \& Pesquies, 1986; Kraemer et al., 1987; Kuel et al., 1978; Stone et al., 1987; Tesch, Colliander, \& Kaiser, 1986). Further, these studies were carried out using different protocols. For instance, in the review of four studies the protocol included one (squat), three (bench press, dead lift, and squat), four (front and back squats, leg press, leg extension) and ten exercises (bench press, leg extension, shoulder press, leg curl, upright rowing, leg press, lat pull down, calf raises, arm curl and hang clean), respectively. The reduced number of studies on the acute physiological responses to heavy resistance exercise, plus the variety of the protocols employed in these studies, creates the necessity to further clarify the acute responses of heart rate, systolic blood pressure, blood lactate levels, and rating of perceived exertion to a whole body workout. The study would examine the acute physiological responses to protocols utilizing different rest interval lengths, as metabolic responses to 
heavy resistance exercise appears to be highly dependent on the length of the rest intervals used (Kraemer et al., 1987). A study of the acute physiological responses to heavy resistance exercise on a whole body workout would help clarify the energy pathways utilized. In addition, information on the relationship between lactate levels and rest interval lengths during heavy resistance exercise would be provided. This in part, would assist to fulfill one of the general considerations on training programs; that is, the training principle of specificity.

\section{Statement of the Problem}

The purpose of the study was to examine the effects of rest interval lengths on acute physiological responses to heavy-resistance exercise.

\section{Approach to the Problem}

The participants of this study were 11 male volunteer subjects from San Jose State University. The group of subjects performed ten-station heavy-resistance exercise protocols using both short and long rest intervals. In the short rest interval protocol each exercise was performed for three sets of $10 \mathrm{RM}$ with a rest interval length of one minute between sets and exercises. The long rest interval protocol included three sets of $10 \mathrm{RM}$ with a rest interval length of three minutes between sets and exercises. Further, the exercises for both protocols were performed in a horizontal workout order. The 
subjects performed the short rest interval length training protocol in the first testing session. The assessment of three physiological variables (heart rate, blood lactate concentration, and systolic blood pressure) and one psychological variable (the rating of perceived exertion) were examined. The measurements for heart rate, blood lactate concentration, and systolic blood pressure were measured on alternate exercises (stations two, four, six, eight, and ten). In addition, the physiological variables in this study were measured at rest and 5-min after post-exercise recovery.

\section{Statement of the Null Hypotheses}

There will be no significant difference in heart rate response between the two training protocols.

There will be no significant difference in the blood lactate concentration between the two training protocols.

There will be no significant difference in the rating of perceived exertion between the two training protocols.

There will be no significant difference in the systolic blood pressure between the two training protocols.

There will be no significant difference in cardiac cost between the two training protocols.

\section{Delimitations of the Study}

This study was delimited to 11 healthy male volunteers aged 20 to 35 years from San Jose State University. All subjects had 
previous experience in heavy-resistance exercise. Furthermore, no attempt was made to control any dietary pattern of the participants. All subjects were free of injury at the time of the study.

\section{Limitations of the Study}

Limitations of this study included the subjects' present training level, pretesting physical activity, nutritional status, genetic makeup, and motivational level.

\section{Assumptions of the Study}

It was assumed that the subjects avoided exhaustive physical activity the day prior to the testing sessions. It was also assumed that the instruments functioned properly and the testers were reliable and objective. In addition it was assumed that the subjects were motivated and followed instructions in order to put forth an optimal effort during the laboratory testing sessions. Finally, it was assumed that the subjects were representative of the population from which they were extracted.

\section{Definition of Terms}

Adenosine Diphosphate (ADP). A complex chemical compound which, when combined with inorganic phosphate (Pi), forms ATP. Adenosine Triphosphate (ATP). A complex chemical compound formed with the energy released from food and is stored in all cells. 
Adenosine Triphosphate-Phosphocreatine System (ATP-PC

System). An anaerobic energy system in which ATP is manufactured when PC is broken down. This system represents the most rapidly available source of ATP for use by muscle. Activities performed at maximum intensity in a period of 10 seconds or less obtain energy (ATP) from this system. The ATP-PC system is also known as the alactacid system (Fox, Bowers, \& Foss, 1988).

Anaerobic Glycolysis (AnG). AnG is referred to as the incomplete chemical breakdown of carbohydrate, also known as the lactic acid system. In this anaerobic energy system the ATP is manufactured when glucose (sugar) is broken down to lactic acid. High intensity efforts requiring one to three minutes draw energy (ATP) primarily from this system (Fox, Bowers, \& Foss, 1988).

Blood Pressure (BP). BP is the driving force that moves the blood through the circulatory system. Systolic pressure is obtained when blood is ejected into the arteries; diastolic pressure indicates dilation of the heart cavities, during which they fill with blood.

Cardiac Cost (CC). CC is defined as the response of the circulatory system to overload and is estimated by the product of systolic blood pressure and submaximal heart rate.

Glycogenesis $(\mathrm{Gg})$. The manufacture of glycogen from glucose.

Glycogenolysis $(\mathrm{Ggl})$. The breakdown of glycogen to glucose.

Glycolysis (Gl). The incomplete chemical break down of glycogen. In aerobic glycolysis, the end product is pyruvic acid; in 
anaerobic glycolysis (lactic acid system), the end product is lactic acid (Karlsson, 1971).

Heart Rate (HR). HR is the number of ventricular contractions as measured by an electrocardiogram (Astrand \& Rodahl, 1986).

Heavy-Resistance Exercise (HRE). Described as a type of exercise which requires the body's musculature to move or attempt to move against some type of opposing force presented by various types of equipment. The most commonly used heavy-resistance exercises are free weights and various weight machines.

Horizontal Workout (HW). HW is defined as a workout in which all of the sets for a given exercise are performed sequentially with rest intervals taken after each exercise and set of exercises.

Lactic Acid (LA). Lactic acid or lactate is a fatiguing metabolite of the lactic acid system resulting from the incomplete breakdown of glucose or glycogen (Fox, Bowers, \& Foss, 1988).

Maximal Voluntary Contraction (MVC). MVC is defined as a maximal load. For instance, the last repetition in a set to failure is a MVC even though the force produced would not be the maximal force possible if the muscle was not partially fatigued.

Metabolic System (MS). A system of biochemical reactions that causes the formation of waste products (metabolites) and the manufacture of ATP; for example, the phosphagen, glycolytic, and oxidative energy systems.

Phosphocreatine (PC). A chemical compound stored in muscle, which when broken down is used to replenish ATP. 
Protocol (P). Protocol refers to either 1-min rest interval between sets and exercises (TP1) or 3-min rest interval between sets and exercises (TP3).

Rating of Perceived Exertion (RPE). The evaluation, by the performer, of the degree of effort or the sensation of physiological effort set forth on a particular physical activity. RPE is determined by the use of a category-ratio scale.

Repetition Maximum (RM). A "X" RM refers to the amount of resistance that allows the performance of " $\mathrm{X}$ " repetitions in good form, with " $\mathrm{X}$ " plus one repetition in good form being impossible (Fleck \& Kraemer, 1987).

Rest intervals (RI). The time assigned for relief; can be placed after each exercise or set of exercises depending on the orientation of the workout.

Specificity of Training (ST). Is a principle underlying construction of a training program for a specific activity and the primary energy system(s) involved during performance.

Steady State (SS). A steady state condition denotes a work situation where oxygen uptake equals the oxygen requirements of the tissues. Heart rate, cardiac output, and pulmonary ventilation attains fairly constant levels (Astrand \& Rodahl, 1986).

Total Body Workout (TBW). The performance of a ten station training protocol designed to stress the major muscle groups of the body. 
Weight Training (WT). Refers to one type of heavy-resistance exercise modality that overloads the muscle(s) to induce a desired training stimuli. In this study WT will be considered synonymous with heavy resistance exercise.

Workout (W). Workout refers to the rest, exercise, and recovery measurements taken during the 1-min or 3-min rest interval training protocol. 


\section{CHAPTER II \\ REVIEW OF LITERATURE}

\section{Introduction}

It has been established that heavy resistance exercise can enhance muscular performance (Anderson \& Kearney, 1982). In addition, it is well supported that the primary energy systems of heavy-resistance exercise are the phosphagen and glycolytic energy systems (Dudley \& Murray, 1982; Fleck \& Kraemer, 1987; Fox, Bowers, \& Foss, 1988; Grimby, Bjorntorp, Fahlen, Hoskins, Hook, Oxhoj, \& Saltin, 1973; Guezennec et al., 1986; Keul et al., 1978; Kraemer, 1983; Kraemer et al., 1987; Stone et al., 1987; Tesch et al., 1986). Substantial increases in blood lactate levels are evident during and immediately after heavy resistance exercise, suggesting that glycolysis makes a significant energy contribution to total energy production (Hurley, Seals, Ehsani, Cartier, Dalsky, Hagberg, \& Holloszy, 1984; Guezennec, et al., 1986; Kraemer et al., 1987; Stone et al., 1987; Tesch et al., 1986).

In addition, strength training may induce changes in the muscle substrate levels. MacDougall, Ward, Sale, and Sutton (1977) reported increased concentrations of ATP, PC, creatine, and glycogen in the triceps brachii muscle of individuals who participated in a five month strength training program. However, the energy systems utilized in a heavy resistance exercise training protocol are currently unknown. How much is the change in LA concentration following a resistive type of exercise with different intensities and should the 
athlete exert extra effort to attain a particular goal in his/her training program are questions which have not been answered. Research shows that the relationship between rating of perceived exertion and LA concentration correspond with the rate of glycogenolytic metabolism during exercise (Noble, Borg, Jacobs, Ceci, \& Kaiser, 1983).

Knowing the phosphagen and glycolytic energy systems' response to a given heavy resistance exercise would help to clarify the adaptative responses which may result from a given training stimulus. A review of the literature was done with the objective of determining the response (rates or levels) of HR, LA, and RPE to heavy resistance exercise and the possible implications of different training protocols on these acute physiological responses.

\section{Background of Energy Metabolism Studies}

It has been demonstrated that an opposite relationship exists between the workload and the PC concentration in the muscle during work (Hultman, Bergstrom, \& Anderson, 1967). One study (Hultman et al., 1967) was performed using needle biopsies in the muscle quadriceps femoris of 27 normal subjects at rest and in connection with work on a bicycle ergometer. The workload in these experiments was $900 \mathrm{kpm}$ and the duration of the work periods was 10 minutes in all of the tests but one, which was five minutes in duration. The results showed that at a very high work load the PC concentration decreases rapidly and, hence, the contractile capacity 
of the muscles also decreased. During heavy work with pronounced decreases in PC, the ATP concentration was also reduced. When the PC level is near zero, the ATP decrease is approximately $40 \%$ of the base value. However, it was reported that after 2-4 minutes of rest the ATP-PC concentration had approached resting levels. They concluded that a considerable oxygen debt without LA formation can be accounted for by breakdown of active phosphate in the working muscles (Hultman et al., 1967).

The speed of LA formation in blood during physical work has been generally accepted as an expression of the existence of anaerobic metabolism (Schwaberger, Pessenhofer, \& Schimid, 1985). Opinion varies regarding the degree of anaerobic metabolism at different work intensities (Pernow \& Wahren, 1962). Margaria, Cerretelli, and Mangili (1964) conducted a study to clarify the mechanism of contraction and the amount of the alactacid component of oxygen debt. They concluded that contraction of alactacid oxygen debt is a fast process requiring 10-30 seconds for completion, while the lactacid debt process is completed in about 40 seconds in the most strenuous exercise. Thus, at the highest work loads (running at $18 \mathrm{~km} / \mathrm{hr}$ with $25 \%$ treadmill inclination) the only energy source available after 40 seconds is the oxidative processes.

Jacobs, Tesch, Bar-Or, Karlsson, and Dotan (1983) studied the contribution of anaerobic glycogenolysis to energy production by measuring intramuscular LA concentration during supramaximal efforts. Lactate levels were determined after 10 and 30 seconds of 
supramaximal exercise (Wingate anaerobic test) in male and female subjects. The LA concentrations averaged 36 and $61 \mathrm{mmol} / \mathrm{kg}$ dry wt after the 10 and 30 seconds exercise bouts, respectively. The male subjects had higher $(\underline{p}<.005)$ LA concentrations and generated higher $(\mathrm{p}<.001)$ power outputs for both exercise bouts than the female subjects. The results showed that pronounced LA accumulation occurs after 10 seconds of supramaximal exercise, suggesting that glycolysis can occur within this time frame. The authors suggested that the conception of being able to perform short bursts of supramaximal exercise solely at the expense of endogenous ATP and PC stores must be reconsidered.

Pernow and Wahren (1962), examined the effect of muscular work with a hand ergometer for 10 and 30 seconds in eight subjects. After performing contractions until exhaustion, the LA concentration in the blood rose significantly from $0.91 \pm 0.25 \mathrm{mM} / \mathrm{L}$ to $2.87 \pm 1.42$ $\mathrm{mM} / \mathrm{L}(\underline{p}<.001)$. One and three minutes after the end of work, the concentration had risen even further. The investigators concluded that a significant rise in LA concentration occurs in the blood during brief work at high intensity.

In another study of energy utilization during intermittent exercise of supramaximal intensity (Margaria, Oliva, Di Prampero, \& Cerretelli, 1969), three subjects were exposed to a run (10 seconds) at $18 \mathrm{~km} / \mathrm{hr}$ at $15 \%$ treadmill inclination. After the resting periods $(10,20$, or 30 seconds) the subjects ran again for 10 seconds, and so on, until exhaustion or steady state was reached. The increase of LA 
concentration in blood due to a 10 second run increased as the rest periods decreased from $3 \mathrm{mg} / 100 \mathrm{ml}$ for a 20 second rest to 11 $\mathrm{mg} / 100 \mathrm{ml}$ for a 10 second rest period. The results suggested that the minimal time of rest at which no LA formation occurred was about 25 seconds, which is the minimal time necessary to eliminate the oxygen debt contracted during the 10 seconds of exercise. Thus, if the recovery period is too short the oxygen debt contracted during exercise can not be paid-back completely (Margaria et al., 1969).

The dynamics of LA accumulation in working muscles was studied in three subjects performing maximal bicycle exercise of 2,6 , and 16 minutes duration by Karlsson and Saltin (1970). From the muscle metabolite determinations, it was concluded that the phosphagens were depleted to the same extent after two to three minutes whether the work load was exhaustive or not. The LA production increased until exhaustion as indicated by muscle tissue and blood lactate concentrations. The authors stated that it seems reasonable to assume that the intensity of the work is a significant factor that stimulates increased glycolytic activity.

\section{Heavy Resistance Exercise and Metabolism}

This review concerns acute metabolic responses to heavy resistance exercise (HRE). HRE relies primarily on the anaerobic energy systems with modest contributions from oxidative metabolism. In a relatively early study Keul et al. (1978) studied 15 experienced weight lifters to determine the effects of WT exercise 
with variable intensity on acute physiological responses. Three exercises (bench press, dead lift, and squat) were utilized in the protocol (two and five minute rest periods between sets and exercises, respectively). The 10 repetitions of WT caused a marked increase in heart rate (150 beats) and only moderate increases in LA concentration $(4 \mathrm{mmol} / \mathrm{L})$. The authors concluded that the energy requirements during WT appeared to be met exclusively through the depletion of the energy-rich phosphates.

Conversely, Tesch et al. (1986) looked at muscle metabolic changes occurring during intense and prolonged, heavy resistance exercise. Their protocol involved five sets of exercises (front and back squats, leg press, and leg extensions) performed until muscle exhaustion (6 to 12 repetitions). The duration of the workout was about $30 \mathrm{~min}$, with an exercise: rest ratio of 1:2. As indicated in Table 1, after exercise muscle ATP, PC, and glycogen levels decreased and creatine, glucose, and LA levels increased $(p<.01)$. Blood LA averaged $17.3 \mathrm{mmol} / \mathrm{kg}$ wet $\mathrm{wt}$ after exercise versus $3.5 \pm 1.6$ $\mathrm{mmol} / \mathrm{kg}$ wet wt before exercise. This data contradicts the suggestion put forth by Keul et al. (1978) which stated that WT is executed solely at the expense of ATP-PC energy stores. In conclusion, heavy resistance exercise of high intensity is associated with a high rate of energy utilization through phosphagen breakdown and activation of glycogenolysis.

The effects of squatting exercise on HR and blood LA levels and rating of perceived exertion (RPE) were studied in five trained and 
TABLE 1

Muscle metabolite concentration before and after exercise

(Tesch et al. 1986)

\begin{tabular}{|c|c|c|c|}
\hline & Pre & Post & Probability \\
\hline ATP & $5.9 \pm 0.3$ & $4.7 \pm 0.7$ & $p<.01$ \\
\hline $\mathrm{PC}$ & $21.3+3.7$ & $10.9+2.5$ & $\mathrm{p}<.001$ \\
\hline Creatine & $12.1 \pm 3.4$ & $23.8+6.0$ & $\mathrm{p}<.001$ \\
\hline Glucose & $0.3 \pm 0.1$ & $3.9 \pm 0.9$ & $p<.001$ \\
\hline Lactate & $3.5 \pm 1.6$ & $17.2+4.6$ & $\underline{p}<.001$ \\
\hline Glycogen & $160 \pm 20$ & $118 \pm 24$ & $\underline{p}<.001$ \\
\hline
\end{tabular}

Note. Values $(\mathrm{mmol} / \mathrm{kg}$ wet $\mathrm{wt})$ are mean $\pm \underline{\mathrm{SD}}$. 
five untrained men (Stone et al. , 1987). The protocol consisted of sets of ten repetitions with five minutes recovery periods until exhaustion. An experimenter assisted by calling out the squatting cadence (one repetition per five sec). In this study voluntary exhaustion was defined as a point at which no more repetitions could be performed. The results (Table 2) showed that at exhaustion the trained subjects had significantly higher heart rates and lactate levels than the untrained subjects. As expected, the trained subjects performed more work (repetitions) at any given intensity. This study suggested that with higher volumes and loading weight training does strongly activates glycolysis and that appropriate weight training may influence anaerobic capacity. An investigation of the effects of successive weight lifting sessions on several hormones and metabolites was performed by Guezennec, Leger, Lhoste Aymonod, and Pesquies, (1986). Eleven males with a beginning maximal free weight bench press of $116.4 \pm 19.2 \mathrm{~kg}$ participated in the study. The training regimen for the first month was as follows: 10 repetitions at $70 \%$ of maximal load or maximal voluntary contraction (MVC), 3-4 sets per exercise with 2.5 min rest interval, 3-4 exercises per session, and 3-4 sessions per week. Typical exercises were supine and incline bench press, leg press, dips, curls, back extensions, and pectoral exercise. In the last eight weeks of the training program the repetitions per set were reduced from 10 to 3 and the relative load was increased from $70 \%$ to $95 \%$ of MVC. The results indicated a significant increase $(\underline{p}<.05)$ in the 
TABLE 2

Heart rate and Lactate levels in five trained and five untrained men after squatting exercise (Stone et al., 1987).

\begin{tabular}{lcc}
\hline & Untrained subjects & Trained subjects \\
Resting HR & $86.4 \pm 1.3$ & $85.8 \pm 7.0$ \\
\hline Exhaustion HR & $172.2 \pm 31.2$ & $182.2 \pm 30.0^{*}$ \\
HR five min after & $114.2 \pm 11.0$ & $115.0 \pm 17.0$ \\
reaching exhaustion & & $1.7 \pm 0.3$ \\
Resting LA level & $1.0 \pm 0.1$ & $10.4 \pm 1.6^{*}$ \\
LA level at exhaustion & $6.1 \pm 0.6$ & $10.2 \pm 1.1^{*}$ \\
LA level five min & $6.3 \pm 1.0$ & \\
after reaching exhaustion & & \\
\hline
\end{tabular}

Note. Values are reported as mean \pm SEM.

$* p<.05$ 
maximal performance of the bench press-exercise test to $135 \pm 27$ kg. LA concentrations increased significantly $(\mathrm{p}<.05)$ from rest to sub-maximal work and maximal work. In addition, glycerol and triglycerides levels increased from rest to work each month of training ( $p<.05)$, while free fatty acid levels (FFA) did not change. The authors concluded that these metabolic responses to weight training indicate that carbohydrate stores are probably the key fuel for the resynthesis of phosphagen stores depleted during heavy exercise.

Unfortunately, in reviewing the current literature, there is only one study that has looked at the acute metabolic responses during the performance of a whole body workout. Kraemer et al., (1987) compared the acute physiological responses of heavy resistance exercise in competitive bodybuilders (BB) and power lifters (PL). The exercise protocol was performed for three sets of $10 \mathrm{RM}$ with a 10 second rest between sets. A 30-60 second rest was taken between exercises to obtain blood samples. The workout called for ten stations (bench press, double leg extension, shoulder press, double leg curl, upright row, leg press, lat pull-down, seated calf raises, arm curl, and hang clean). HR levels, RPE, and plasma LA concentrations significantly increased above resting levels, during the workout in both groups of subjects. HR during exercise ranged from 160 to190 bpm, LA concentration rose from 5.0 to $21.0 \mathrm{mmol} / \mathrm{l}$, and the RPE increased from 2.5 to 9.0 on a ratio scale of 10 . PL 
exhibited less of an increase in HR and LA responses and a higher increase in RPE than $\mathrm{BB}$. The results demonstrated that larger increases in plasma lactate concentrations resulted when rest was further reduced during a whole body exercise protocol. Thus, these results suggest that the metabolic responses to heavy resistance exercise may be highly dependent on the length of the rest periods used.

Gordon, Russell, Kruger, and Cilliers (1985) studied eight young adults males during a WT session. The protocol called for three sets of 15 repetitions of nine exercises performed at a work cadence of 15 repetitions per minute with one-minute recovery intervals. The workload was 50,75 and $100 \%$ for the first, second and third sets, respectively. The maximal oxygen uptake rate (VO2max) of the subjects was $3.89 \pm 0.61 \mathrm{~L} / \mathrm{min}$ and the $\mathrm{HRmax}$ was $192 \pm 7 \mathrm{bpm}$. The strenuous nature of WT was evident from the Borg's RPE scale rating of $17.1 \pm 0.9$. The average HR during exercise was $138 \pm 16$ bpm (72\% $\pm 7 \%$ of HRmax) and oxygen uptake rate (VO2) was $1.26 \pm$ $0.33 \mathrm{~L} / \min (32 \pm 8 \%$ of treadmill $\mathrm{VO} 2 \max )$. The LA concentrations increased from $1.2 \pm 0.5$ to $7.0 \pm 1.3 \mathrm{mmol} / \mathrm{L}$ during WT. It is interesting to note that although the values for average HR, LA concentration, and RPE were fairly high, the oxygen cost was reported to be a mere $32 \pm 8 \%$ of treadmill VO2max.

Hurley, Seals, Ehsani, Cartier, Dalsky, Hagberg, and Holloszy (1984) looked at the effects of high-intensity strength training on cardiovascular function. Thirteen untrained adult males were 
subjects in a Nautilus training protocol that included 14 exercises. Subjects moved as fast as possible from one station to the next during the training which consisted of one set of $8-12 R M$ workload performed three to four times per week for a total of 16 weeks. Oxygen uptake rate, HR, LA levels, and RPE from a single session (acute response) of the Nautilus workout were measured. The results showed that oxygen uptake rate during the Nautilus workout represented $45 \pm 2 \%$ of the subjects' maximal oxygen uptake rate while walking at $4.0 \mathrm{mph}$ on a treadmill (TM). The HR during the Nautilus exercise was equal to $80 \%$ of HR Reserve (HRR) compared to a HR of only $45 \%$ of HRR during the TM walking. The RPE was $125 \%$ higher during Nautilus exercise and the blood LA concentrations was 16-fold higher after the Nautilus exercise compared to walking. In an attempt to explain the disproportionate increase in HR relative to the VO2max, the authors suggested that sympathetic stimulation probably played an important role in the increase in HR without a parallel increase in oxygen uptake rate.

The energy cost of circuit weight training was studied by Wilmore, Parr, Ward, Vodak, Barstow, Pipes, Grimditch, and Leslie (1978). The method included two groups of 20 young adult subjects who performed three circuits of 10 stations, using a work (30 second) to rest (15 second) ratio of $2: 1$ for a total exercise time of 22.5 minutes. The workload was equal to $40 \%$ of a one repetition maximum (1RM). The results showed the same trend as the previous study as the male subjects were working above $70 \%$ of HRmax but 
below $45 \%$ of VO2max. The female subjects worked at a value above $80 \%$ of HRmax but below $50 \%$ of VO2max.

The relationship of plasma volume change to intensity of weight lifting was studied in 15 young adult males by Collins, Cureton, Hill, and Ray (1989). Weight lifting was performed at 40, 50,60 , and $70 \%$ of $1 \mathrm{RM}$ and consisted of completing three circuits of four exercises (bench press, bent-over row, arm curl, and parallel squat) with ten repetitions of each exercise performed over a 30 second period followed by 30 seconds of rest. Table 3 summarizes the results of this study. The authors concluded that plasma volume decreases linearly in relation to the intensity ( $\% 1 \mathrm{RM})$ of weight lifting and that the relationship is similar to that reported for dynamic, low-resistance exercise such as cycling or running

\section{Summary}

The theoretical basis of energy metabolism generally agrees that heavy resistance exercise is a physical activity that relies primarily on the anaerobic energy pathways. It is suggested that the very early stages of exercise WT is primarily supported first, by the phosphagen (ATP-PC) system and second, by the anaerobic glycolysis system (Dudley \& Murray, 1982; Fleck \& Kraemer, 1987; Fox, Bowers, \& Foss, 1988; Grimby, Bjorntorp, Fahlen, Hoskins, Hook, Oxhoj, \& Saltin, 1973; Guezennec et al., 1986; Keul et al., 1978; Kraemer, 1983; Kraemer et al., 1987; Stone et al., 1987; Tesch et al., 1986). 
TABLE 3

Maximal oxygen uptake rate (VO2), blood lactate (LA), and heart rate (HR) responses during and following four intensities of weight training (Collins et al. 1989)

\section{INTENSITY (\% of 1 RM)}

\begin{tabular}{lcccc} 
& $40 \%$ & $50 \%$ & $60 \%$ & $70 \%$ \\
\hline VO2 & & & & \\
$(\mathrm{L} / \mathrm{min})$ & $1.31 \pm 0.04$ & $1.50 \pm 0.07$ & $1.72 \pm 0.07$ & $1.78 \pm 0.08$ \\
$\mathrm{LA}$ & & & & \\
$(\mathrm{mmol} / \mathrm{L})$ & $6.5 \pm 0.5$ & $8.7 \pm 0.5$ & $9.4 \pm 0.6$ & $12.0 \pm 0.6$ \\
$\mathrm{HR}$ & & & & \\
$(\mathrm{bpm})$ & $124 \pm 4$ & $134 \pm 4$ & $148 \pm 5$ & $161 \pm 4$ \\
Plasma & & & & \\
volume & & & & \\
$(\%)$ & $-7.7 \pm 0.8$ & $-10.7 \pm 0.8$ & $-12.1 \pm 0.9$ & $-13.9 \pm 0.8$ \\
\hline
\end{tabular}

Note. The values for VO2 and HR represent $33-47 \%$ and $63-82 \%$ of treadmill-determined VO2max and HRmax, respectively. All values are expressed as $M \pm S E M$. 
In addition, there are some studies supporting that lipolysis is also activated, although this is controversial. Phosphagen stores deplete very rapidly, but approximately $70 \%$ of phosphagen restoration is completed after 30 seconds and $100 \%$ within 3 to 5 minutes of rest.

Various studies looked at acute energy metabolism responses to exercise. In general, the vast majority examined variables such as VO2, HR, RPE, and blood LA concentration (Table 4). The methodology employed included treadmill testing, telemetry electrocardiographic recording, rating of perceived exertion, and lactate analysis. Among the studies reviewed, the methodology of "the experimental workouts" utilized included variety of training protocols ranging from a single exercise (squat) test to three (bench press, dead lift, and squat), four (front squat and back squat, leg press, and leg extension) and ten exercises. The latter was the only study that looked at a whole body workout of ten exercises (bench press, leg extension, shoulder press, leg curl, upright rowing, leg press, lat pull-down, calf raise, arm curl, and hang clean). The results indicated that phosphate metabolism and glycolytic metabolism are utilized during a WT workout. The magnitude of the increase in LA concentrations appears to be greater in training protocols utilizing shorter rest intervals. In conclusion, the metabolic responses to WT appear to depend on the nature of the stimuli; that is, the intensity of the workload, type and amount of exercises performed in the workout, number of sets and repetitions, and length of the rest intervals. The literature was deficient in finding a study featuring a 
TABLE 4

Summary of the results of acute physiological responses to heavy resistance exercise

Investigators

\begin{tabular}{lll}
$\mathrm{N}$ & $\mathrm{La}^{*}$ & $\mathrm{HR}{ }^{*}$ \\
$(\mathrm{mmol} / \mathrm{L})$ & $(\mathrm{bpm})$ & $\mathrm{RPE} *$ \\
\hline
\end{tabular}

\begin{tabular}{lccrcc} 
Hurley et al. & $(1984)$ & 13 & 16.0 & 155 & 18 \\
Guezennec et al. & $(1986)$ & 11 & 5.8 & - & - \\
Kraemer et al. & $(1987)$ & 17 & 21.0 & 180 & $9 \mathrm{a}$ \\
Stone et al. & $(1987)$ & 10 & 10.4 & 182 & 18 \\
Tesch et al. & $(1986)$ & 9 & 17.3 & - & - \\
Collins et al. & $(1989)$ & 15 & 12.0 & 161 & - \\
Gordon et al. & $(1985)$ & 8 & 7.0 & 138 & 17 \\
Keul et al. & $(1978)$ & 15 & 4.5 & 120 & - \\
\hline
\end{tabular}

Note. LA, RPE, and HR levels are the mean values during the workout.

a This study used a 10 points rating scale.

* The results are statistically significant from pre-exercise values. 
whole body workout with short ( $<1$ minute) and long ( $>3$ minutes) rest intervals. Therefore, it is justified to investigate the acute physiological responses of a heavy resistance exercise protocol with short ( $<1$ minute) and long ( $>3$ minutes) rest intervals. 


\section{CHAPTER III}

\section{METHODS}

\section{Introduction}

This chapter includes information about the subjects, experimental design, measurements and methods, training protocol, and statistical analyses.

\section{$\underline{\text { Subjects }}$}

The subjects were males aged 20 to 35 years. The participants in this study consisted of 11 volunteers from the San Jose State University. The subjects were currently involved in heavy resistance exercise. The subjects completed a health screening questionnaire (Appendix. A) and signed a human subjects informed consent form (Appendix B) before participating in the study.

\section{Experimental Design}

The study required the subjects to report to the laboratory on three different occasions: (1) to set the workload (1RM test) for the resistance exercise training sessions, (2) to perform the training session with short rest intervals (Appendix C), and (3) to perform the training session with long rest intervals (Appendix D). The estimated time for the subjects to complete the testing procedures was approximately two hours. In the first session the subjects' one repetition maximum (1RM) was determined for each of the 10 training exercises. In the second and third testing sessions the subjects performed the training protocol with short and long rest 
intervals, respectively. The WT sessions (horizontal workout) included the use of free-weight and machine apparatus with a workout of three sets of 10 exercises with alternated rest intervals (short and long) between sets and exercises. In addition, during the WT sessions, heart rate, rating of perceived exertion, blood lactate concentration, and systolic blood pressure were determined after every other set of exercises.

\section{Measurements and Methods}

One Repetition Maximum (1RM)

The subjects were tested with free weights and/or Universal equipment on every exercise of the training protocol to determine the 1RM. This procedure was done with the purpose of determining the workload for each muscle group (exercise) at each station. Then, the load for the training sessions was set at a 10 repetition maximum (10 RM) load that was determined in base of a percent (70-80\%) of the 1RM (Kraemer et al., 1987).

\section{Training Sessions}

The experimental workout order and type of exercise stations was (1) bench press (Olympic barbell), (2) leg Press (Universal Machine), (3) lat pull-down (Universal machine), (4) leg curl (Universal machine), (5) Military Press (Universal Machine), (6) upright rowing (barbell), (7) leg extension (Universal machine), (8) triceps push-down (Universal machine), (9) calf raise (Olympic 
barbell), and (10) arm curl (barbell). Each exercise was performed for three sets of $10 \mathrm{RM}$ with one minute rest intervals for the training protocol number one (Appendix $C$ ) and three minute rest intervals for the training protocol number two (Appendix D). Both training sessions had the characteristics of a horizontal workout. The subjects performed the training session with short rest intervals first. The training sessions were preceded by a standardized warm-up that included stretching, calisthenics, and the formal activity to be performed during the workout (Appendix E). Also a standardized cool-down period followed after the WT sessions, which included exercises from the formal activity, calisthenics, and stretching exercises (Appendix F).

\section{Heart Rate Assessment}

Submaximal heart rate responses were measured during the standardized workouts. Subjects were continuously monitored via a standard V5-manubrium electrode lead placement to a Narco physiograph ECG recorder. The ECG monitoring system was used to measure heart rate and observe the rhythmic pattern of the subject's heart throughout the training sessions. Heart rate was recorded during the last 10 to 15 seconds of the last set of repetitions of every other exercise. Also, heart rate was measured before and five minutes after exercise. 
Blood lactate determination

Fingertip blood samples were obtained using a syringepet after every other exercise (exercise stations $2,4,6,8$, and 10) to determine blood LA concentration. In addition, blood collections using a syringepet were obtained before and five minutes after exercise. Blood LA was analyzed by a Yellow Springs Incorporated (YSI) lactate analyzer (YSI Model 23L). The YSI lactate analyzer was calibrated every two blood samples. The sample collections, handling, and lactate determination was done in accordance with the guidelines set forth in the YSI model 23L Lactate Analyzer Instruction Manual (1988).

\section{Blood Pressure Assessment}

Blood pressure measurements were obtained from the left arm of the subjects before, during, and after exercise. Blood pressure was monitored manually before and five minutes after exercise. In addition, blood pressures were determined immediately after exercise at stations two, four, six, eight, and ten. Blood pressure determination was performed manually using an sphygmomanometer and stethoscope.

\section{$\underline{\text { Rating of Perceived Exertion }}$}

The RPE during exercise was measured using the category-ratio scale (Noble et al., 1983). This subjective rating of the intensity of exercise was obtained immediately after the last set of repetitions of 
every other exercise by having the subjects rate their perceived exertion from a table (ten point-category ratio scale).

\section{Statistical Analyses}

The statistical evaluation of the data included descriptive statistics for heart rate, lactate, and systolic blood pressure levels for

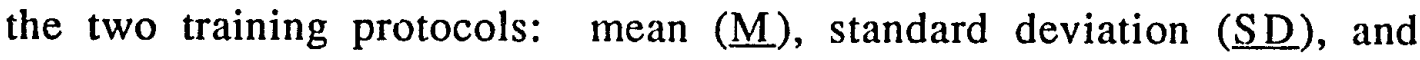
range at rest; after exercise stations $2,4,6,8$, and 10; and after recovery from the workout. These descriptive statistics were also calculated for rating of perceived exertion after exercise stations 2,4 , 6, 8, and 10. Dependent $\mathbf{t}$-tests were used to compare heart rate, lactate levels, and systolic blood pressure levels from the two training protocols at rest. Two-way analyses of variance were used to compare heart rate, lactate, and systolic blood pressure levels from the two training protocols at rest; after exercise stations $2,4,6$, 8 , and 10; and after recovery from the workout. Also, a two-way analysis of variance was used to compare rating of perceived exertion after exercise stations $2,4,6,8$, and 10 . Dependent $\underline{t}$-tests and one-way analyses of variance with Tukey post hoc tests were used as follow-up procedures on the significant main effects found in the two-way analyses of variance. 


\section{CHAPTER IV}

\section{ANALYSIS OF DATA}

\section{Introduction}

This chapter is organized in several sections as follows: level of significance, analysis of data, summary of findings, discussion of findings, conclusions, weaknesses, recommendations for future research, and summary.

\section{Level of Significance}

A probability level (p) of .05 or less was selected as the level of statistical significance for all the analyses of data.

\section{Analysis of data}

Eleven healthy male subjects were tested in the study. There were no missing values. Table 5 summarizes the physical characteristics of the subjects.

Table 6 summarizes the maximal strength and mean training loads at each station of the training protocol. The mean and standard deviation workloads are reported in pounds.

Table 7 summarizes the mean training protocol performed by the subjects. Some of the subjects were unable to perform three sets of 10 repetitions at each exercise station due to the severity of the workout with the short rest intervals.

Table 8 summarizes the results of paired $\underline{t}$-tests for training 
TABLE 5

Physical Characteristics of the Subjects

\begin{tabular}{lccc} 
& $\begin{array}{c}\text { Age } \\
(\mathrm{yr})\end{array}$ & $\begin{array}{c}\text { Body weight } \\
(\mathrm{kg})\end{array}$ & $\begin{array}{c}\text { Height } \\
(\mathrm{cm})\end{array}$ \\
\hline $\mathrm{M}$ & 27.6 & 80.6 & 180.4 \\
$\mathrm{SD}$ & 4.4 & 10.1 & 7.4 \\
Range & $22.0-34.0$ & $68.2-96.4$ & $167.6-195.5$ \\
& & & \\
\hline $\mathrm{N}=11$ & & &
\end{tabular}


TABLE 6

Maximal Strength and Mean Training Loads at each Station

\begin{tabular}{lrr}
\hline Exercise station & Strength $(1 \mathrm{RM})$ & \multicolumn{1}{l}{ Training load } \\
\hline Bench press & $217.27 \pm 49.26$ & $150.27 \pm 34.47$ \\
Leg press & $421.82 \pm 57.76$ & $293.86 \pm 40.05$ \\
Lat pulldown & $177.27 \pm 31.96$ & $123.18 \pm 21.99$ \\
Leg curl & $80.22 \pm 13.58$ & $54.72 \pm 9.51$ \\
Military press & $119.54 \pm 20.24$ & $83.18 \pm 14.32$ \\
Upright rowing & $109.54 \pm 22.74$ & $76.36 \pm 15.50$ \\
Leg extension & $161.81 \pm 25.62$ & $112.50 \pm 17.21$ \\
Tricep pressdown & $90.45 \pm 16.80$ & $62.72 \pm 11.42$ \\
Calf raises & $215.90 \pm 20.71$ & $150.45 \pm 13.50$ \\
Arm curl & $103.63 \pm 20.50$ & $71.81 \pm 14.01$ \\
\hline
\end{tabular}

Workloads are reported in $1 b, M \pm S D$.

$\mathrm{N}=11$ 


\section{TABLE 7}

Mean Training Protocol Performed by the Subjects

\begin{tabular}{llll}
\hline & & \multicolumn{2}{l}{ Sets } \\
Exercise station & 1 & 2 & 3 \\
\hline Bench Press & 10 & 10 & 9.3 \\
Leg Press & 10 & 10 & 10 \\
Lat pulldown & 10 & 10 & 10 \\
Leg curl & 10 & 10 & 9.8 \\
Military press & 10 & 10 & 9.5 \\
Upright rowing & 10 & 10 & 9.8 \\
Leg extension & 10 & 10 & 10 \\
Tricep pressdown & 10 & 10 & 10 \\
Calf raises & 10 & 10 & 10 \\
Arm curl & 10 & 9.3 & 8.2 \\
\hline
\end{tabular}


TABLE 8

Results of Paired t-tests for Training Protocols with 1 and 3 minutes Rest Intervals Lengths

\begin{tabular}{|c|c|c|c|c|c|}
\hline Variable & $\mathrm{TP}$ & Mean & SE & Diff & t-value \\
\hline bl rest & TP1 & 1.46 & .16 & & \\
\hline bl rest & TP3 & 1.58 & .25 & -.12 & -.43 \\
\hline bl leg press & TP1 & 5.33 & .48 & & \\
\hline bl leg press & TP3 & .99 & .30 & 1.8 & $7.62 *$ \\
\hline bl leg curl & TP1 & 6.37 & .43 & & \\
\hline bl leg curl & TP3 & 4.07 & .34 & 2.30 & $9.74 *$ \\
\hline bl upright row & TP1 & 8.09 & .58 & & \\
\hline bl upright row & TP3 & 5.06 & .42 & 3.02 & $12.90 *$ \\
\hline bl tricep pressdown & TP1 & 8.90 & .67 & & \\
\hline bl tricep pressdown & TP3 & 4.74 & .53 & 4.15 & $12.21 *$ \\
\hline bl arm curl & TP1 & 8.42 & .74 & & \\
\hline bl arm curl & TP3 & 4.24 & .43 & 4.18 & $8.96^{*}$ \\
\hline bla recovery & TP1 & 8.15 & .73 & & \\
\hline bla recovery & TP3 & 3.50 & .27 & 4.65 & $6.53 *$ \\
\hline rpe leg press & TP1 & 3.90 & .44 & & \\
\hline rpe leg press & TP3 & 3.45 & .68 & .45 & .70 \\
\hline rpe leg curl & TP1 & 5.45 & .80 & & \\
\hline rpe leg curl & TP3 & 3.63 & .58 & 1.81 & $2.89 *$ \\
\hline
\end{tabular}


Table 8 continued:

\begin{tabular}{|c|c|c|c|c|c|}
\hline Variable & $\mathrm{TP}$ & Mean & SE & Diff & $\underline{\mathrm{t} \text {-value }}$ \\
\hline rpe upright row & TP1 & 6.09 & .72 & & \\
\hline rpe upright row & TP3 & 3.72 & .51 & 2.36 & $4.49 *$ \\
\hline rpe tricep pressdown & TP1 & 6.09 & .61 & & \\
\hline rpe tricep pressdown & TP3 & 3.45 & .49 & 2.63 & $4.70^{*}$ \\
\hline rpe arm curl & TP1 & 7.54 & .59 & & \\
\hline rpe arm curl & TP3 & 3.81 & .58 & 3.72 & $5.31 *$ \\
\hline sbp rest & TP1 & 124.8 & 1.98 & & \\
\hline sbp rest & TP3 & 123.5 & 1.83 & 1.27 & .56 \\
\hline sbp leg press & TP1 & 141.8 & 3.03 & & \\
\hline sbp leg press & TP3 & 138.5 & 3.27 & 3.27 & .84 \\
\hline sbp leg curl & TP1 & 141.1 & 3.27 & & \\
\hline sbp leg curl & ТP3 & 140.7 & 2.53 & .45 & .12 \\
\hline sbp upright row & TP1 & 145.3 & 3.19 & & \\
\hline sbp upright row & TP3 & 138.7 & 4.15 & 6.63 & 1.40 \\
\hline sbp tricep pressdown & $\mathrm{TP} 1$ & 140.0 & 3.07 & & \\
\hline sbp tricep pressdown & TP3 & 130.0 & 3.56 & 10.0 & $2.48 *$ \\
\hline sbp arm curl & TP1 & 143.5 & 3.81 & & \\
\hline sbp arm curl & TP3 & 136.4 & 3.33 & 7.09 & 1.98 \\
\hline sbp recovery & TP1 & 127.7 & 2.50 & & \\
\hline sbp recovery & TP3 & 124.4 & 2.68 & 3.27 & 1.10 \\
\hline
\end{tabular}


Table 8 continues:

\begin{tabular}{|c|c|c|c|c|c|}
\hline Variable & $\mathrm{TP}$ & Mean & SE & Diff & t-value \\
\hline hr rest & TP1 & 71.49 & 3.29 & & \\
\hline hr rest & TP3 & 63.74 & 2.75 & 7.74 & $2.53^{*}$ \\
\hline hr leg press & TP1 & 131.07 & 5.58 & & \\
\hline hr leg press & TP3 & 120.63 & 4.79 & 10.43 & $2.61 *$ \\
\hline hr leg curl & TP1 & 143.75 & 5.19 & & \\
\hline hr leg curl & TP3 & 125.21 & 5.52 & 18.53 & $8.97 *$ \\
\hline hr upright row & TP1 & 159.27 & 5.49 & & \\
\hline hr upright row & TP3 & 125.74 & 5.34 & 33.52 & $8.45^{*}$ \\
\hline hr tricep pressdown & TP1 & 148.56 & 5.87 & & \\
\hline hr tricep pressdown & TP3 & 120.39 & 6.89 & 28.17 & $5.99 *$ \\
\hline hr arm curl & TP1 & 159.68 & 6.02 & & \\
\hline hr arm curl & TP3 & 126.12 & 6.66 & 33.55 & $8.98 *$ \\
\hline hr recovery & TP1 & 101.55 & 4.71 & & \\
\hline hr recovery & TP3 & 77.77 & 3.92 & 23.78 & $6.14 *$ \\
\hline cc rest & TP1 & 8923.20 & 425.73 & & \\
\hline cc rest & TP3 & 7888.74 & 390.43 & 1034.45 & 2.93 \\
\hline cc leg press & TP1 & 18605.40 & 897.75 & & \\
\hline cc leg press & TP3 & 16752.69 & 885.79 & 1852.70 & 2.05 \\
\hline cc leg curl & TP1 & 20320.38 & 916.90 & & \\
\hline cc leg curl & TP3 & 17659.19 & 962.92 & 2661.19 & 3.76 \\
\hline cc upright row & TP1 & 23228.20 & 1094.30 & & \\
\hline cc upright row & TP3 & 17464.68 & 916.80 & 5763.51 & 7.90 \\
\hline
\end{tabular}

$* \mathrm{p}<.05$ 
Table 8 continues:

\begin{tabular}{lccrrr} 
Variable & TP & Mean & SE & Diff & t-value \\
\hline cc tricep pressdown & TP1 & 20871.76 & 1092.28 & & \\
cc tricep pressdown & TP3 & 15763.58 & 1195.93 & 5108.18 & 5.41 \\
& & & & & \\
cc arm curl & TP1 & 22877.43 & 973.30 & & \\
cc arm curl & TP3 & 17275.49 & 1102.96 & 5601.94 & 7.08 \\
& & & & & \\
cc recovery & TP1 & 12983.52 & 686.91 & & \\
cc recovery & TP3 & 9684.76 & 546.07 & 3298.76 & 6.29 \\
\hline
\end{tabular}

Note. Values for blood lactate (bl), heart rate (hr), systolic blood pressure ( $\mathrm{sbp}$ ), and cardiac cost (cc) are expressed in $\mathrm{mmol} / \mathrm{L}$, beats per minute, $\mathrm{mm} \mathrm{Hg}$, and $\mathrm{bpm} \times \mathrm{mm} \mathrm{Hg}$, respectively. Values for rate of perceived exertion (rpe) represent Borg's category-ratio scale.

$\mathrm{TP} 1=$ training protocol with one minute rest intervals

TP3 $=$ training protocol with three minute rest intervals

$* \underline{p}<.05$ 
protocols with one and three minutes rest interval lengths. The summary tables for each of the ANOVAs are presented in Appendix L-1 through Appendix L-5.

Table 9 summarizes the results for blood lactate concentration during the training protocols. Two way analysis of variance revealed significant differences between training protocols, $\underline{F}(1,10)=122.32$ $\underline{p}<.01$ and across the workouts, $\underline{F}(6,60)=59.14 \underline{p}<.01$ as well as the interaction between protocols and workouts, $\underline{F}(6,60)=27.55 \underline{p}<$ .01 . One-way analyses of variance on the workouts revealed that all exercise stations (lactate concentration fluctuated between 3.5 and $8.9 \mathrm{mmol} / \mathrm{L}$ ) were significantly different from resting values for the short $(1.4 \mathrm{mmol} / \mathrm{L})$ and long $(1.5 \mathrm{mmol} / \mathrm{L})$ training protocols. Tukey post hoc analyses detected significant differences between exercise stations four and six $(8.0 \mathrm{mmol} / \mathrm{L})$ for the short training protocol and between stations four $(4.0 \mathrm{mmol} / \mathrm{L})$ and $\mathrm{six}(5.0 \mathrm{mmol} / \mathrm{L})$ for the long training protocol. A dependent t-test revealed that resting values of $1.4 \mathrm{mmol} / \mathrm{L}$ for the short protocol and $1.5 \mathrm{mmol} / \mathrm{L}$ for the long protocol were not significantly different, $\underline{\mathrm{t}}(10)=-.43 \mathrm{p}<.01$. All exercise and recovery lactate levels were significantly higher during the short training protocol than the long training protocol.

Table 10 summarizes the results for heart rate during the training protocols. Two-way analysis of variance covarying for differences in resting heart rates revealed significant differences between the two protocols, $\underline{F}(1,9)=83.85 \underline{p}<.01$ and across the 
TABLE 9

Responses of Blood Lactate Concentration During the Training Protocols

\begin{tabular}{llllllll}
\hline Rest & 2 & 4 & 6 & 8 & 10 & $\operatorname{Rec}$ \\
\hline
\end{tabular}

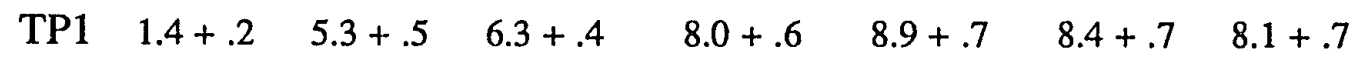

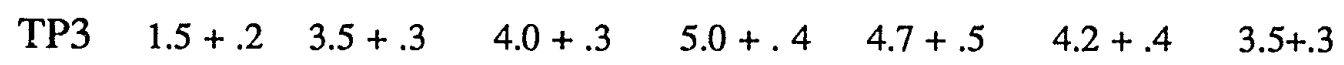

Note. Values are $\mathrm{mmol} / \mathrm{L}$ and represent the $\mathrm{M} \pm \mathrm{SEM}$.

Two-way analysis of variance revealed significant $(\underline{p}<.05)$

differences between the two protocols, $\underline{F}(1,10)=122.32 \underline{p}<.01$ and across the workouts, $\mathrm{F}(6,60)=59.14 \mathrm{p}<.01$ as well as the interaction between protocols and workouts, $\mathrm{E}(6,60)=27.55 \mathrm{p}<.01$. One-way analyses of variance on the workouts revealed that significant differences existed across both TP1 and TP3. Tukey post hoc analyses indicated that all of the exercise and recovery blood lactate levels were significantly higher than resting blood lactate levels for both training protocols. Also, significant differences between exercise stations four and six for the TP1 and between stations four and six for the TP3 were noted. A dependent t-test revealed that resting values were not significantly different, $\underline{\mathbf{t}}(10)=$ $-.43 \underline{p}<.01$. However, all exercise and recovery blood lactate levels were significantly higher during TP1 than TP3. 
TABLE 10

Heart Rate Responses During the Training Protocols

\begin{tabular}{lrrrrrrr}
\hline & Rest & 2 & 4 & 6 & 8 & 10 & Rec \\
\hline TP1 & & & & & & & \\
M & 71.4 & 131.0 & 143.7 & 159.2 & 148.5 & 159.6 & 101.5 \\
SEM & 3.2 & 5.5 & 5.1 & 5.4 & 5.8 & 6.0 & 4.7 \\
\hline TP3 & & & & & & & \\
M & 63.7 & 120.6 & 125.2 & 125.7 & 120.3 & 126.1 & 77.7 \\
SEM & 2.7 & 4.7 & 5.5 & 5.0 & 6.8 & 6.6 & 3.9 \\
\hline
\end{tabular}

Note. Values are beats per minute (bpm) and represent the $M \pm S E M$. Two-way analysis of variance covarying for differences in resting heart rate revealed significant $(p<.05)$ differences between the two protocols, $\underline{F}(1,9)=83.85 \underline{p}<.01$ and across the workouts, $\underline{F}(5,50)=71.36 \underline{p}<.01$ as well as the interaction between protocols and workouts, $E(5,50)=$ $6.43 \mathrm{p}<.01$. A dependent $\mathrm{t}$-test revealed that resting heart rates in TP1 were significantly higher than in TP3, $\underline{t}(10)=$ $2.53 \mathrm{p}<.01$. One-way analyses of variance on the workouts revealed significant differences across both training protocols. Tukey post hoc analyses indicated that all exercises and recovery heart rates were significantly higher than the resting heart rates for both TP1. Also significant differences in heart rates existed between stations two and four and between stations four and six in the short training protocol. Also all exercise and recovery HR values were significantly higher during TP1 than TP3. 
workouts, $\underline{F}(5,50)=71.36 \underline{p}<.01$ as well as the interaction between protocols and workouts, $\underline{\mathrm{F}}(5,50)=6.43 \mathrm{p}<.01$. A dependent t-test revealed that the mean resting value of $71.4 \mathrm{bpm}$ for the TP1 was Tukey post hoc analyses revealed that all of the exercise and recovery heart rate responses were significantly higher than the resting heart rate responses for both training protocols. Also significant differences between stations two $(131.0 \mathrm{bpm})$ and four (143.7 bpm) and four (143.7 bpm) and six (159.2 bpm) in the short protocol were noted. Moreover, all exercise and recovery heart rate responses were significantly higher during TP1 than TP3. Examination of the significant interaction revealed that heart rate responses during both protocols exhibited a similar overall pattern, although heart rates dropped more sharply during recovery (101.5 vs. $77.7 \mathrm{bpm}$ ) in the long training protocol (TP3).

Table 11 summarizes the results for rating of perceived exertion during both training protocols. Two-way analysis of variance revealed significant differences between the two protocols, $\underline{F}(1,10)=22.26 \mathrm{p}<.01$ and across the workouts, $\underline{F}(4,40)=8.73 \mathrm{p}<$ .01 as well as a significant interaction between protocols and workouts, $\underline{F}(4,40)=7.02 \underline{p}<.01$. One-way analyses of variance on the workouts revealed that significant differences existed across TP1, but not TP3. Tukey post hoc analyses on TP1 revealed significant differences between the rating of perceived exertion for stations two (3.9) and four (5.4). however, in stations four 5.4 vs. 3.6 , $\underline{\mathrm{t}}(10)=$ 
TABLE 11

Rating of Perceived Exertion During the Training Protocols

\begin{tabular}{cccccc}
\hline & 2 & 4 & 6 & 8 & 10 \\
\hline $\mathrm{TP} 1$ & $3.9 \pm .4$ & $5.4 \pm .8$ & $6.0 \pm .7$ & $6.0 \pm .6$ & $7.5 \pm .6$ \\
\hline $\mathrm{TP} 3$ & $3.4 \pm .7$ & $3.6 \pm .6$ & $3.7 \pm .5$ & $3.4 \pm .5$ & $3.8 \pm .6$ \\
\hline
\end{tabular}

Note. Values represent Noble's ten point scale and are $M \pm$ SEM. Two-way analysis of variance revealed significant $(\underline{p}<.05)$ differences between the two protocols, $\underline{F}(1,10)=22.26 \underline{p}<.01$ and across the workouts, $\underline{F}(4,40)=8.73 \mathrm{p}<.01$ as well as a significant interaction between protocols and workouts, $\underline{E}(4,40)=7.02 \underline{p}<.01$. One-way analyses of variance indicated that significant differences existed across TP1, but not TP3. Tukey post hoc analyses revealed significant differences between stations two and four in TP1. Dependent $\mathrm{t}$-tests revealed significantly higher RPE values at stations four, $\underline{\mathrm{t}}(10)=2.89 \mathrm{p}<.01$, six, $\underline{\mathrm{t}}(10)=4.49 \underline{\mathrm{p}}<.01$, eight, $\underline{\mathrm{t}}(10)=4.70$ $\mathrm{p}<.01$, and ten, $\underline{\mathrm{t}}(10)=5.31 \mathrm{p}<.01$ for TP1. RPE values of station two were not significantly different between the workouts. 
$2.89 \mathrm{p}<.01$, six 6.0 vs. $3.7, \underline{\mathrm{t}}(10)=4.49 \mathrm{p}<.01$, eight 6.0 vs. $3.4, \underline{\mathrm{t}}$ $(10)=4.70 \underline{\mathrm{p}}<.01$, and ten 7.5 vs. $3.8, \underline{\mathrm{t}}(10)=5.31 \mathrm{p}<.01$, there were significantly higher RPE values in TP1 than in TP3. The significant interaction was primarily due to the fact that the rating of perceived exertion rose more sharply between stations eight (6.0) and ten (7.5) in TP1 than TP3. Blood lactate concentrations were significantly correlated to rating of perceived exertion (RPE) during station six in the short rest interval protocol $(\underline{r}=.83)$ and station ten during the long rest interval protocol $(\underline{r}=.78)$. Heart rate responses were significantly correlated to rating of perceived exertion during station six in the short rest interval protocol $(\underline{r}=.62)$ and station eight during the long rest interval protocol $(\underline{r}=.88)$.

Table 12 summarizes the responses of systolic blood pressure (SBP) during the workouts for the two training protocols. Two-way analysis of variance revealed significant differences across the workouts, $\underline{F}(6,60)=21.56 \underline{p}<.01$. One-way analyses of variance and Tukey post hoc tests revealed significant differences between rest $(124.8 \mathrm{~mm} \mathrm{Hg}$ ) and the exercise stations (SBP fluctuated between 140.0 and $145.3 \mathrm{~mm} \mathrm{Hg}$ throughout the exercise stations) in the short protocol. In addition there were differences between rest $(123.5 \mathrm{~mm} \mathrm{Hg}$ ) and the exercise stations (SBP fluctuated between 130.0 and $140.7 \mathrm{~mm} \mathrm{Hg}$ throughout the exercise stations) in the long protocol. Tukey post hoc analyses revealed no significant differences between exercise stations in TP1. However, significant differences 
TABLE 12

Systolic Blood Pressure Responses During the Training Protocols

\begin{tabular}{|c|c|c|c|c|c|c|c|}
\hline & Rest & 2 & 4 & 6 & 8 & 10 & $\operatorname{Rec}$ \\
\hline \multicolumn{8}{|l|}{ TP1 } \\
\hline M & 124.8 & 141.8 & 141.1 & 145.3 & 140.0 & 143.5 & 127.7 \\
\hline SEM & 1.9 & 3.0 & 3.2 & 3.1 & 3.0 & 3.8 & 2.5 \\
\hline \multicolumn{8}{|l|}{ TP3 } \\
\hline M & 123.5 & 138.5 & 140.7 & 138.7 & 130.0 & 136.4 & 124.4 \\
\hline SEM & 1.8 & 3.2 & 2.5 & 4.1 & 3.5 & 3.3 & 2.6 \\
\hline
\end{tabular}

Note . Values are $\mathrm{mm} \mathrm{Hg}$ and represent the $\mathrm{M} \pm$ SEM.

Two-way analysis of variance revealed significant $(\mathrm{p}<.05)$

differences across the workouts, $\underline{F}(6,60)=21.56 \mathrm{p}<.01$.

One-way analyses of variance on the workouts revealed

significant differences between rest and the exercise stations

in both TP1 and TP3. Tukey post hoc analyses indicated that

all exercise systolic blood pressure responses were

significantly higher than resting and recovery responses in

both TP1 and TP3. 
between stations six $(138.7 \mathrm{~mm} \mathrm{Hg})$ and eight $(130.0 \mathrm{~mm} \mathrm{Hg})$ in $\mathrm{TP} 3$ were found. There was not a significant interaction between protocols and workouts.

Table 13 summarizes the results of cardiac cost (CC) during the workout for the two training protocols. Two-way analysis of variance covarying for differences in resting cardiac cost between short and long protocols $(8,923.2$ vs. $7,888.7 \mathrm{bpm} \mathrm{x} \mathrm{mm} \mathrm{Hg}$, respectively) revealed significant differences between the two protocols, $\underline{\mathrm{F}}(1,9)=30.18 \underline{\mathrm{p}}<.01$ and across the workouts, $\underline{\mathrm{F}}(5,50)=$ $84.04 \mathrm{p}<.01$ as well as a significant interaction between protocols and workouts, $\underline{F}(5,50)=6.54 \underline{p}<.01$. One-way analyses of variance revealed significant differences across the workouts in both training protocols. Tukey post hoc analyses indicated that significant differences existed between rest $(8,923.2 \mathrm{bpm} \times \mathrm{mm} \mathrm{Hg})$ and all exercise stations (CC fluctuated between 18,605.4 and 23,228.2 bpm $\mathrm{x} \mathrm{mm} \mathrm{Hg}$ throughout the exercise stations) as well as, between rest $(8,923.2 \mathrm{bpm} \times \mathrm{mm} \mathrm{Hg})$ and recovery $(12,983.5 \mathrm{bpm} \times \mathrm{mm} \mathrm{Hg})$ in the short protocol. The long protocol exhibited significant differences between rest $(7,888.7 \mathrm{bpm} \times \mathrm{mm} \mathrm{Hg})$ and exercise stations two $(16,752.6 \mathrm{bpm} \times \mathrm{mm} \mathrm{Hg})$, four $(17,659.1 \mathrm{bpm} \times \mathrm{mm} \mathrm{Hg})$, six $(17,464.6 \mathrm{bpm} \times \mathrm{mm} \mathrm{Hg})$, eight $(15,763.5 \mathrm{bpm} \times \mathrm{mm} \mathrm{Hg})$, and ten $(17,275.4 \mathrm{bpm} \times \mathrm{mm} \mathrm{Hg})$. Dependent $\underline{\mathrm{t}}$-tests revealed significant differences between TP1 and TP3 for rest $(8,923.2$ vs. $7,888,7 \mathrm{bpm} \times$ $\mathrm{mm} \mathrm{Hg}) \underline{\mathrm{t}}(10)=2.93 \underline{\mathrm{p}}<.01$, exercise stations four $(20,320.3$ vs. 
TABLE 13

Cardiac Cost During the Training Protocols

\begin{tabular}{lrrrrrrr}
\hline & Rest & \multicolumn{1}{c}{2} & 4 & \multicolumn{1}{c}{6} & 8 & 10 & Rec \\
\hline TP1 & & & & & & & \\
M & $8,923.2$ & $18,605.4$ & $20,320.3$ & $23,228.2$ & $20,871.7$ & $22,877.4$ & $12,983.5$ \\
SEM & 425.7 & 897.7 & 916.9 & $1,094.3$ & $1,092.2$ & 973.3 & 686.9 \\
& & & & & & & \\
\hline TP3 & & & & & & & \\
M & $7,888.7$ & $16,752.6$ & $17,659.1$ & $17,464.6$ & $15,763.5$ & $17,275.4$ & $9,684.7$ \\
SEM & 390.4 & 885.7 & 962.9 & 916.8 & $1,195.9$ & $1,102.9$ & 546.0 \\
\hline
\end{tabular}

Note . Values are $\mathrm{bpm} \times \mathrm{mm} \mathrm{Hg}$ and represent $\mathrm{M} \pm \mathrm{SEM}$.

Two-way analysis of variance covarying for differences in resting cardiac cost revealed significant $(\underline{p}<.05)$ differences between the two protocols, $\underline{F}(1,9)=30.18 \underline{p}<.01$ and across the workouts, $\underline{F}(5,50)=84.04 \underline{p}<.01$ as well as the interaction between protocols and workouts, $\underline{E}(5,50)=6.54 \underline{p}$ $<.01$. One-way analyses of variance and Tukey post hoc analyses revealed significant differences between rest and all exercise stations and between rest and recovery in TP1. Oneway analyses of variance and Tukey post hoc analyses revealed significant differences between rest and all the exercise stations in TP3. Dependent $\underline{t}$-tests revealed significant differences between protocols at rest, $\underline{t}(10)=2.93$ $\underline{p}<.01$; exercise stations four, $\underline{\mathrm{t}}(10)=3.76 \mathrm{p}<.01$, six, $\underline{\mathrm{t}}(10)$ $=7.90 \underline{\mathrm{p}}<.01$, eight, $\underline{\mathrm{t}}(10)=5.41 \mathrm{p}<.01$, and ten, $\underline{\mathrm{t}}(10)=7.08$ $\mathrm{p}<.01 ;$ and recovery, $\underline{\mathrm{t}}(10)=6.29 \mathrm{p}<.01$. 
$17,659.1 \mathrm{bpm} \times \mathrm{mm} \mathrm{Hg}), \underline{\mathrm{t}}(10)=3.76 \mathrm{p}<.01$, six $(23,228.2$ vs. $17,464.6 \mathrm{bpm} \times \mathrm{mm} \mathrm{Hg}), \underline{\mathrm{t}}(10)=7.90 \mathrm{p}<.01$, eight $(20,871.7$ vs. $15,763.5 \mathrm{bpm} \times \mathrm{mm} \mathrm{Hg}), \underline{\mathrm{t}}(10)=5.41 \mathrm{p}<.01$, and ten $(22,877.4$ vs. $17,275.4 \mathrm{bpm} \times \mathrm{mm} \mathrm{Hg}), \underline{\mathrm{t}}(10)=7.08 \mathrm{p}<.01$, and recovery $(12,983.5$ vs. $9,684.7 \mathrm{bpm} \times \mathrm{mm} \mathrm{Hg}), \underline{\mathrm{t}}(10)=6.29 \mathrm{p}<.01$. The significant interaction appeared to be primarily due to changes in cardiac cost at exercise station six where a substantial increase was observed during TP1, but not during TP3.

\section{Summary of Findings}

The two-way analysis of variance for blood lactate concentrations during exercise resulted in significant differences between protocols, across workouts, and an interaction between protocols and workouts. One-way analyses of variance revealed significant differences between rest and all exercise stations during both the short and long rest interval protocols. In addition, the Tukey post hoc analyses revealed significant differences between stations four and six for the short and long rest interval protocols. Dependent $\underline{t}$-tests revealed that TP1 elicited higher blood lactate concentrations than TP3 during all the workout measurements as well as during recovery.

Resting heart rate was significantly different between the two protocols. A two-way analysis of variance covarying for the differences in resting heart rates revealed significant differences between the two protocols and across workouts as well as a 
significant interaction of protocols and workouts. One-way analyses of variance revealed significant differences across both workouts. Tukey post hoc analyses indicated that all exercise and recovery heart rates were significantly higher than resting heart rates for both TP1 and TP3. Tukey post hoc analyses also revealed significant differences between stations two and four and between stations four and six in the short protocol. Also, dependent t-tests showed the heart rate responses at rest, during all the workout measurements, and during recovery were significantly higher during TP1 than during TP3.

Two-way analysis of variance for the rating of perceived exertion revealed significant differences between the two protocols and across the workouts as well as a significant interaction between protocols and workouts. One-way analyses of variance and Tukey post hoc analyses revealed significant differences between stations two and four in TP1. In addition, dependent t-tests showed that the rating of perceived exertion at exercise stations four, six, eight, and ten were significantly higher during TP1 than during TP3.

Two-way analysis of variance for systolic blood pressure revealed significant differences across the workouts. One-way analyses of variances and Tukey post hoc analyses revealed that resting values were significantly lower than all of the exercise values for both TP1 and TP3. In addition, the analyses showed that systolic blood pressure decreased significantly between stations six and eight in TP3. A dependent $\underline{t}$-test revealed that systolic blood pressure was 
significantly lower at station eight in TP3 compared to station eight in TP1.

The cardiac cost $(\mathrm{CC}=$ systolic blood pressure $\mathrm{x}$ heart rate $)$ at rest for the two protocols was significantly different. A two-way analysis of variance covarying for differences in resting cardiac costs revealed significant main effects between the protocols and across the workouts, as well as a significant interaction of protocols by workouts. One-way analysis of variance and Tukey post hoc analyses revealed that the cardiac costs during all exercise measurements were significantly higher than during rest and recovery in TP1. Similarly, during the long protocol the cardiac costs were significantly higher after all exercise stations than at rest. In addition, dependent t-tests showed that cardiac cost was significantly higher at rest during TP1 compared to TP3 and also significantly higher during exercise at stations four, six, eight, and ten as well as during recovery.

\section{Discussion of Findings}

The purpose of this study was to examine the effects of rest interval length on acute physiological and psychological responses to heavy-resistance exercise. After the data was gathered and analyzed for the 11 recreational weight lifters, most of the variables analyzed were significantly different between the short and long training protocol (TP1 elicited greater responses than TP3). A dependent ttest revealed that resting heart rate values before TP1 were 
significantly higher than resting heart rate values before TP3. This may have occurred since the subjects knew that the training protocol with short rest intervals was considerably more severe to perform than the longer protocol and also, because the short protocol was to be performed in the first training session. Therefore, this may have induced some nervousness and state of uncertainty since the subjects knew that they were going to be encouraged to perform the complete workout.

The subjects in this study had substantial increases in blood lactate, heart rate, systolic blood pressure, and rating of perceived exertion levels comparable to increases reported by other investigators (Collins et al., 1989; Gordon et al., 1985; Hurley et al., 1984; Kraemer et al., 1987; Kuel et al., 1976; Stone et al., 1987). For instance, in this study blood lactate levels increased to $8.9 \pm .66$ $\mathrm{mmol} / \mathrm{L}$ (one subject peaked at $13.4 \mathrm{mmol} / \mathrm{L}$ ) during the short protocol. These results are above the $7.0 \mathrm{mmol} / \mathrm{L}$ reported by Gordon et al. (1985), $5.8 \mathrm{mmol} / \mathrm{L}$ reported by Guezennec et al. (1986), $4.5 \mathrm{mmol} / \mathrm{L}$ reported by Keul et al. (1978), and slightly below the results of Stone et al. (1987) who reported $10.4 \mathrm{mmol} / \mathrm{L}$ for trained and $6.1 \mathrm{mmol} / \mathrm{L}$ for untrained subjects. It is important to mention that during Stone's study the subjects performed squat repetitions until exhaustion (defined as the point where the subjects could not perform another repetition). In contrast with the former study, Kraemer et al. (1987) reported increases from 5.0 to 21.0 $\mathrm{mmol} / \mathrm{L}$ for competitive body builders and power lifters. Among the 
reasons for the incredible increases in blood lactate concentration might be due to shorter ( 30 to 60 seconds) rest intervals, the inclusion of structural exercises (bench press and hang clean), and the national training status of the subjects in Kraemer's study. In light of the mentioned results, this study suggested that with higher volumes and loading, resistance training strongly activates glycolysis and that appropriate resistance training methods may influence anaerobic capacity.

As for heart rate, in this study mean values of $159.6 \pm 6.0 \mathrm{bpm}$ were recorded for the last exercise stations of the short protocol. In addition, during the short rest interval protocol peaked values of $182.9 \mathrm{bpm}$ were observed. The review of literature showed that during weight lifting exercises heart rate reached values of 120.0 bpm (Keul et al., 1976), 138.0 bpm (Gordon et al., 1985), 155 bpm (Hurley et al., 1984), 161.0 bpm (Collins et al., 1989), 180.0 bpm (Kraemer et al., 1987), and $182.8 \mathrm{bpm}$ (Stone et al., 1987). Interestingly, the heart rate values of this study were similar to the rate values reported by Collins et al., (1989) when working at $70 \%$ of a 1RM. This suggested that this study had a similar intensity with relation to the one repetition maximum (1RM) and that HR responses in part depended on the number of exercises and the repetitions performed during the workout. Hempel and Wells (1985) reported values in percent of maximal $\mathrm{HR}$ that averaged $77.5 \%$ during a 14 station circuit training workout. In this study the subjects reached values of $82.9 \%$ of the mean predicted maximal HR. 
The mean rating of perceived exertion (RPE) recorded in this study was $7.5 \pm .6$ with a peak value of 10 rating points for the short protocol and $3.8 \pm .6$ with a peak value of 9 rating points for the long protocol. In the only other study that used a ten point rating scale, Kraemer et al. (1987) reported RPE values ranging from two to 10. In a study were a 20 point scale was used, Gordon et al. (1985) found values as high as 18 points. In this study a mean value of 7.5 would represent a workout labeled "very strong" for the short protocol and 3.8 would represent a workout "moderate" to "somewhat strong" for the long protocol. This ratio of "moderate" intensity for the long training protocol suggested that this type of protocol may not be appropriate for inducing development of anaerobic work capacity. From a practical standpoint it is interesting to report that in this study the RPE was significantly related to heart rate in both the short and long protocols ( $\underline{r}=.88$ and $\underline{r}=.62$, respectively), and to blood lactate concentration in both the short and long protocols $(\underline{r}=.83$ and $\underline{r}=.78$, respectively). This observation would support the possible use of a category-ratio scale to monitor the perceptual responses of the subjects during heavy-resistance exercise.

Mean systolic blood pressure (SBP) was $145.3 \pm 3.1 \mathrm{~mm} \mathrm{Hg}$ for the short training protocol and $140.7 \pm 2.5 \mathrm{~mm} \mathrm{Hg}$ for the long training protocol. These results are below the cardiovascular responses $(152 \pm 8 \mathrm{~mm} \mathrm{Hg})$ reported by Hurley et al. (1984) in which older $(44 \pm 1 \mathrm{yr})$ and untrained subjects were used. This study used younger $(27.6 \pm 1.3 \mathrm{yr})$ subjects with some recreational weight 
training experience. This would suggest that younger and better trained individuals while working out at a similar workload, stress the cardiovascular system to a lesser degree.

The cardiac cost (CC) in this study peaked during the short protocol at $23,228.2 \pm 1,090.3 \mathrm{bpm} \times \mathrm{mm} \mathrm{Hg}$ and $17,659.1 \pm 962.9$ bpm $\times \mathrm{mm} \mathrm{Hg}$ during the long protocol. In light of the above results, the $\mathrm{CC}$ during the short protocol increased 2.6-fold with respect to the resting level in order to cope with the demands of this type of training. The cardiac cost for the long protocol increased 2.2-fold in relation to the resting values. The lower cardiac costs observed during the long protocol suggests that this protocol may be more appropriate in programs attempting to minimize myocardial workload.

\section{Conclusions}

Within the limits of this study the following conclusions were made:

(1) There were significantly greater increases in blood lactate concentrations during the short training protocol compared to the long training protocol.

(2) There were significantly greater increases in heart rate responses during the short training protocol compared to the long training protocol. 
(3) There were significantly greater increases in rating of perceived exertion during the short training protocol compared to the long training protocol.

(4) There were significantly greater increases in systolic blood pressure during the short training protocol compared to the long training protocol.

(5) There were significantly greater increases in cardiac cost during the short training protocol compared to the long training protocol.

(6) Since resistance training strongly activates glycolysis, training which uses a short rest interval may improve anaerobic capacity more than training which uses a long rest interval.

(7) A training protocol with long rest intervals and training loads of $70 \%$ of $1 \mathrm{RM}$ or less may not be appropriate for development of anaerobic capacity.

(8) A training protocol with long rest intervals may be appropriate in programs designed to minimize myocardial workload.

\section{Weaknesses}

The subjective assessment of the rating of perceived exertion by the subjects may have been somewhat conservative, as the overall physiological responses, particularly during the short rest interval protocol, appeared to indicate rather stressful cardiovascular and metabolic work demands. Furthermore, it appeared that the exercise technique for the calf muscles (barbell over the shoulders) 
was not the most appropriate exercise to find a true reflection of this muscle group's strength since considerable stress was also being placed on the back. The extensors of the back muscles appeared to be less strong than the gastrocnemius and soleous; therefore, the back muscles fatigued earlier. A better exercise would have been the "seated calf raise." Finally, motivation and training status may have played an important role in testing (setting 1RM), as well as during the actual performance of the training protocols.

\section{Recommendations for Future Research}

Within the limits of this study the following recommendations were made.

(1) Future research should examine the responses of physiological and psychological variables with different levels of intensity and rest interval durations, such as a higher percent of $1 \mathrm{RM}$ with longer rest intervals ( $>5 \mathrm{~min}$ ).

(2) It is also suggested that data from elite athletes using similar training protocols would add additional information to the body of knowledge of heavy-resistance exercise.

(3) It is also suggested that cardiovascular and metabolic responses from younger and older populations would add additional information to the body of knowledge of heavy-resistance exercise.

(4) Future research on acute physiological and psychological responses to heavy-resistance exercise on female subjects should be examined. 
62

References 
American College of Sports Medicine (1986). Guidelines for exercise testing and prescription (3rd ed). Philadelphia: Lea \& Febiger. Anderson, T. , \& Kearney, J. T. (1982). Effects of three resistance training programs on muscular strength and absolute and relative endurance. Research Quarterly for Exercise and Sports, 53(1), 1-7.

Astrand, I. , Astrand, P. O. , Christensen, E. H. , \& Hedman, R. (1960). Intermittent muscular work. Acta Physiologica Scandinavica, $\underline{48}, 448-453$.

Astrand, P. O. , \& Rodahl, K. (1986). Textbook of work physiology: physiological bases of exercise (3rd ed). New York: McGraw-Hill.

Collins, M. A. , Cureton, K. J. , Hill, D. W. , \& Ray, C. A. (1989). Relation of plasma volume change to intensity of weight lifting. Medicine and Science and Sports and Exercise, 21(2), 178-185.

Duddley, G. A. (1988). Metabolic consequences of resistive-type exercise. Medicine and Science in Sports and Exercise, 20(5), 158-161.

Dudley, G. A. , Murray, T. F. (1982). Energy for sport. National Strength and Conditioning Association Journal, 4(3), 14-15.

Fleck, S. J. , \& Kraemer, W. J. (1987). Designing Resistance Training Programs. Illinois: Human Kinetics Books.

Fox, I. L. , Bowers, R. W. , \& Foss, M. L. (1988). The physiological basis of physical education and athletics. Philadelphia: Saunders College Publishing. 
Gordon, N. F. , Russell, H. M. S. , Kruger, P. E. , \& Cilliers, J. F. (1985).

Thermoregulatory responses to weight training. International Journal of Sports Medicine, $\underline{6}(3)=145-150$.

Grimby, G. , Bjorntorp, P. , Fahlen, M. , Hoskins, T. A. , Hook, O. , Oxhoj, O. , \& Saltin, B. (1973). Metabolic effects of isometric training. Scandinavian Journal of Clinical Laboratory of Investigation, $\underline{31}$, $301-305$.

Guezennec, Y. , Leger, L. , Lhoste, F. , Aymonod, M. , \& Pesquies, P. C. (1986). Hormone and metabolite response to weight-lifting training sessions. International Journal of Sports Medicine, $\underline{7}(2)$, $100-105$.

Hultman, E. , Bergstrom, J. , \& Mc Lennan Anderson, N. (1967). Breakdown and resynthesis of phosphorylcreatine and adenosine triphosphate in connection with muscular work in man.

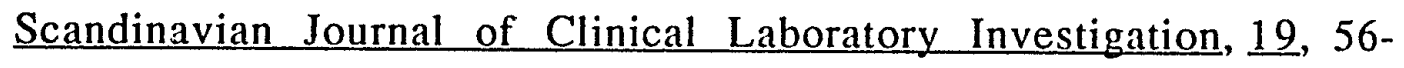
66.

Jacobs, I. , Tesch, P. A. , Bar Or, O. , Karlsson, J. , \& Dotan, R. (1983).

Lactate in human skeletal muscle after 10 and $30 \mathrm{~s}$ of supramaximal exercise. Journal of Applied Physiology, 55(2), 365-367.

Jones, N. L. , McCartney, N. , \& McComas, A. J. (1986). Human muscle power. Illinois: Human Kinetics Publishers.

Karlsson, J. (1971). Lactate and phosphagen concentrations in working muscle of men. Acta Physiologica Scandinavica, (suppl. 358), $1-72$. 
Karlsson, J. , \& Saltin, B. (1970). Lactate, ATP, and CP in working muscles during exhaustive exercise in man. Journal of Applied Physiology, 29(5), 598-602.

Keul, J. , Haralambie, G. , Bruder, M. , \& Gottstein, H. J. (1978). The effects of weight lifting exercise on heart rate and metabolism in experienced weight lifters. Medicine and Science in Sports, 10(1),13-15.

Kraemer, W. (1983). Exercise prescription in weight training: A needs analysis. National Strength and Conditioning Association Journal, 5(1), 64-65.

Kraemer, W. J. , Noble, B. J. , Clark, M. J. , \& Culver B. W. (1987). Physiologic responses to heavy-resistance exercise with very short rest periods. International Journal of Sports Medicine, $\underline{8}(4)$, 247-252.

Margaria, R. , Cerretelli, P. , \& Mangili, F. (1964). Balance and kinetics of anaerobic energy release during strenuous exercise in man. Journal of Applied Physiology, 19(4), 623-628.

Margaria, R. , Oliva, R. D. , Di Prampero, P. E. , \& Cerretelli, P. (1969). Energy utilization in intermittent exercise of supramaximal intensity. Journal of Applied Physiology, 26(6), 752-756.

Noble, B. J. , Borg G. A. V. , Jacobs, I. , Ceci, R. , \& Kaiser, P. (1983). A category-ratio perceived exertion scale: relationship to blood and muscle lactates and heart rate. Medicine and Science in Sports and Exercise, 15(6), 523-528. 
Pernow, B. , \& Wahren, J. (1962). Lactate and pyruvate formation and oxygen utilization in the human forearm muscles during work of high intensity and varying duration. Acta Physiologica Scandinavica, $\underline{56}, 267-285$.

Schwaberger, G. , Pessenhofer, H. , \& Schmid, P. (1985). Relevance of the lactate determination in sports medicine. Wien Med Wochenscher, 135(9-10), 234-241.

Stone, M. H. , O' Braynt, H. , Garhammer, J. , McMillan, J. , \& Rozenek, R. (1982). Theoretical model of strength training. National Strength and Conditioning Association Journal, 4(4), 36-39.

Stone, M. H. , Pierce, K. , Godsen, R. , Wilson, D. , Blessing, D. , Rozenek, R. , \& Chromiak, J. (1987). Heart rate and lactate levels during weight-training exercise in trained and untrained men. The Physician and Sportsmedicine, 15(5), 97-105.

Tesch, P. A. (1988). Skeletal muscle adaptations consequent to longterm heavy resistance exercise. Medicine and Science in Sports and Exercise, 20(5), 132-134.

Tesch, P. A. , Colliander, E. B. , \& Kaiser, P. (1986). Muscle metabolism during intense, heavy-resistance exercise. European Journal of Applied Physiology, 55, 362-366.

Wilmore, J. H. , Parr, R. B. , Ward, P. , Vodak, P. A. , Barstow, T. J. , Pipes, T. V. , Grimditch, G. , \& Leslie, P. (1978). Energy cost of circuit weight training. Medicine and Science in Sports, 10(2), $75-78$. 
Yellow Springs Incorporated Model 23L: Lactate Analyzer

Instruction Manual. (1988). Yellow Springs Instrument Co., Inc. Yellow Springs, Ohio. 
APPENDIX A

PRE-EXERCISE TESTING HEALTH STATUS QUESTIONNAIRE 


\section{SAN JOSE STATE UNIVERSITY \\ DEPARTMENT OF HUMAN PERFORMANCE \\ PRE-EXERCISE TESTING HEALTH STATUS QUESTIONNAIRE}

Name

Occupation

Work Address

Home

Address

Personal

Physician

Age: $\quad$ yr

Height: $\mathrm{ft}$ and in
Phone

Home

Phone

Physician's

Phone

Weight:

lb

Does the body weight indicate: a gain , a loss , or no change , in the past year? How many pounds?

Joint-Muscle Status (Check areas which you currently have problems).

Joint Areas

( ) Wrists

( ) Elbows

( ) Shoulders

( ) Upper spine \& neck

( ) Lower spine

( ) Hips

( ) Knees

( ) Ankles

( ) Feet

( ) Other

( ) Other

Muscle Areas 
( ) Arm

( ) Shoulders

( ) Chest

( ) Upper back \& neck

( ) Abdominal region

( ) Lower back

( ) Buttocks

() Thighs

( ) Lower leg

( ) Feet

( ) Other

( ) Other

Medical History: (Check if you had any of the following conditions).

\begin{tabular}{|c|c|c|}
\hline Currently & \multicolumn{2}{|c|}{ Previously } \\
\hline ( ) & ( ) & High blood pressure \\
\hline ( ) & ( ) & Heart disease or dysfunction \\
\hline ( ) & ( ) & $\begin{array}{l}\text { Chest discomfort-especially with } \\
\text { exertion }\end{array}$ \\
\hline ( ) & ( ) & Rheumatic fever \\
\hline ( ) & ( ) & Ankle swelling \\
\hline ( ) & ( ) & Unusual shortness of breath \\
\hline ( ) & ( ) & Lightheadedness or fainting \\
\hline ( ) & ( ) & Emotional disorders \\
\hline ( ) & ( ) & Orthopedic Problems \\
\hline () & ( ) & Peripheral circulation disorder \\
\hline () & ( ) & Lung disease or dysfunction \\
\hline () & () & Arthritis or gout \\
\hline () & ( ) & Edema \\
\hline () & () & Epilepsy \\
\hline () & ( ) & Multiple sclerosis \\
\hline () & () & High blood cholesterol or \\
\hline () & () & triglyceride levels \\
\hline () & () & Acute infection \\
\hline & & Diabetes or blood sugar \\
\hline
\end{tabular}




$\begin{array}{lll} & & \text { level abnormality } \\ \text { ( ) } & \text { ( ) } & \text { Anemia } \\ \text { () } & \text { () } & \text { Hernias } \\ \text { () } & \text { ( ) } & \text { Thyroid dysfunction } \\ \text { () } & \text { () } & \text { Pancreas Dysfunction } \\ \text { () } & \text { () } & \text { Liver dysfunction } \\ \text { () } & \text { () } & \text { Kidney Dysfunction } \\ \text { () } & \text { ( ) } & \text { Neural dysfunction } \\ \text { () } & \text { () } & \text { Others } \\ \text { () } & \text { () } & \\ \text { () } & \text { () } & \end{array}$

Physical Examination History:

Date of your last physical examination

Physical problems noted at that time

When was the last time your resting electrocardiogram was evaluated?

Was it normal? If no, what was abnormal about it?

When was the last time you had your electrocardiogram evaluated during an exercise stress test? What heart rate did you reach during this exercise?_ Was the electrocardiogram normal? Yes ( ) No ( ). If no, what was abnormal about it ?

Has a physician ever made any recommendations relative to limiting your levels of physical exertion? Yes ( ) No ( ). If yes, what limitations were recommended? 
Current Medication Usage: (list the drug name and the condition being managed)

Medication Condition

Physical Perceptions: Indicate any unusual sensations or perceptions. Check if you have recently experienced any of the following during or soon after physical activity (PA) or during sedentary periods (SED).

PA SED

( ) ( )

( )

( )

(

( ) ( ) Muscle cramping

( ) ( ) Muscle pain

( ) ( ) Joint pain
PA SED

( ) ( ) Lightheadedness

( ) ( ) Loss of balance

( ) ( ) loss of coordination

() () Extreme weakness

() ( ) Numbness

( ) ( ) Mental confusion

() () Nausea

( ) ( ) Other

Family History: Check if any of your blood relatives such as grandparents, parents, aunts, uncles, brothers, and sisters, have or had any of the following.

( ) Heart disease at what age?

( ) Heart attacks or strokes at what age?

( ) Sudden death at what age?

( ) Elevated blood cholesterol or triglycerides level. 

( ) High blood pressure.
( ) Diabetes.

Current Habits: Check any of the following if they are characteristic of your current habits.

( ) Occupation is physically demanding.

( ) Occupation is emotionally stressful and/or hectic.

( ) In your leisure, you regularly do manual garden or yard work.

( ) In your leisure, you regularly go for long walks.

( ) You frequently ride a bicycle.

( ) You engage in an exercise program of three times per week. If so, what does this consist of ?

( ) You smoke tobacco: ( ) cigarettes ( ) cigars ( ) pipes.

Number per day ( packs, cigars, or pipeful).

Exercise Training Experience: Check if you ever have had an exercise program issued to you by any of the following professionals.

\section{( ) Exercise Physiologist}

( ) Physical Educator

( ) Strength and Conditioning Coach

() Personal Trainer

( ) Other

If so, do you know the intensity duration and frequency of your exercise program? 
Do you have experience with free weights and/ or machine equipment? (Circle the number identifying the best answer)

$\begin{array}{llllll}1 & 2 & 3 & 4 & 5\end{array}$

Strongly agree agree somewhat Disagree Strongly disagree

If so, how much experience do you have practicing with this equipment?_ yr months,

What would you say was the orientation of your training protocol?

( ) Muscular strength.

( ) Muscular endurance.

( ) Muscular hypertrophy.

( ) other

How did you work to achieve the desired goal? 
APPENDIX B INFORMED CONSENT FORM 
RESPONSIBLE INVESTIGATOR: Hugo F. Quevedo

TITLE OF PROTOCOL: Study to Examine the Effects of Rest Intervals on Acute Physiological Responses to Heavy Resistance Exercise.

Invitation to Participate

You are invited to participate in a study that will investigate the effects of rest intervals on acute physiological responses to heavy resistance exercise. The study requires the subjects to report to the laboratory on three different occasions. The training protocol and the testing procedures of this study will take place in SPX 202-A.

\section{$\underline{\text { Requirements for Selection }}$}

You will be selected as a participant in this study, if you are a healthy male aged 20 and $35 \mathrm{yr}$, who is currently involved in resistance exercise. Before participation, an exercise physiologist will review your health history questionnaire and if satisfactory, you will be asked to participate in the tests described below.

\section{Explanation of Testing Procedures}

One Repetition Maximum (1RM) (approximately 30 minutes)

This test will involve the measurement of maximal strength for each one of the ten exercises to be performed in the training 
sessions. A tentative workload will be agree upon between the subject and the investigator and by trial-and-error the 1RM will be established. If more than five attempts are needed to determine the $1 R M$, that particular test will be discarded and a new test will be performed on a future date. Once the $1 \mathrm{RM}$ is known, the 10RM workload will be established in the same manner as in previously published research.

Heavy Resistance Exercise (approximately 2:00 hrs)

After the performance of a standardized warm-up period, you will be engaged in a free weight training session. The protocol will include, three sets of ten exercises, with rest intervals of either one or three minutes, respectively. Blood lactate levels, heart rate and, rate of perceived exertion will be measured. After the workouts a cool down period will follow.

\section{Heart Rate Measurement}

Your heart rate will be monitored with a V5 lead attached to your chest, during the workout. The electrocardiogram will show the rhythmical pattern of your heart, and heart rate will be recorded during the last ten seconds of the third set for each exercise.

\section{Blood Lactate Levels}

The blood lactate levels will be determined before, during, and after the HRE protocol, by drawing a fingertip blood sample. After a finger prick with a sterile needle, blood will be drawn by a sterile syringepet for analysis of lactate concentration in the blood. 


\section{Blood Pressure Measurements}

Blood pressure will be estimated manually by using a sphygmomanometer and stethoscope. This measurements will take place before, during, and five minutes after exercise and will reflect the cardiocirculatory load.

\section{$\underline{\text { Rate of Perceived Exertion }}$}

You will rate the subjective intensity of the workout several times throughout the training sessions. You will be ask to identify your effort by grading the exertion of the work performed on a category scale after every other exercise.

\section{Discomforts and Risks}

\section{Maximal Strength and Training Protocol}

During the performance of these tests you may experience, an increase in blood pressure; body temperature; breathing rate; elevated heart rate; profuse sweating; and possible fatigue. Approximately 24 to 48 hours after the workout, you may experience some temporary muscle soreness.

\section{Analysis of Blood Lactate Levels}

Lactate levels will be assessed by drawing blood from the fingertips (micro-puncture). This process will be repeated several 
times during the testing protocols. The discomfort and risks involved are minimal, although your fingertips may be sore for a short while.

Benefits from Participation in the Study

You will benefit from this study by receiving an evaluation of your maximal body strength (1RM) for all the major muscle groups. The evaluation will also, give you feedback related to your relative muscular strength (10RM). You may, also obtain information about the three physiological responses to the training protocol (heart rate, lactate levels, and blood pressure). In addition if you decide to engage in this type of activity, the prescription of a heavy resistance exercise will be offered to you. As for the population, they will benefit from this study by improved scientific information for designing resistance exercise programs.

\section{Assurance of Confidentiality}

The data drawn from this investigation may be used for scientific purposes at conferences and/or meetings. Your name will not be disclosed unless your express consent is granted prior to the publication and/or presentation of the data.

\section{Withdrawal from the Study}

You may withdraw your consent and discontinue your participation in this investigation at any time (including during the testing procedures) without prejudice. Testing will be supervised and conducted by Dr. Craig J. Cisar, Certified Exercise Test Technologist (ACSM) and Certified Strength and Conditioning 
Specialist (NSCA), and Hugo F. Quevedo, a Human Performance Graduate Student from San Jose State University.

\section{Inquiries}

If you have any question about participating in this investigation or regarding to the test procedures feel free to ask us, at any time. Please call Hugo (415-358-9120) and I will be more than happy to answer your questions. Any complaints or additional questions about the procedures may be presented to Dr. Craig Cisar, (408-924-3018). Questions or complaints about research subjects rights, or in the event of research-related injury, may be directed to Dr. Serena Stanford, Associate Academic Vice President for Graduates Studies and Research (408-924-2480).

\section{Consent}

After reading the above, I agree that:

(a) my consent is given voluntarily without being coerced.

(b) I understand the procedures explained to me by the investigators.

(c) I will participate in the study.

(d) I know the risks and/or discomforts involved with the study. 
(e) I can withdraw from the study or from any part of it, without any prejudice to my relationship with San Jose State University.

(f) I understand that the data are confidential but may be published or presented without revealing my identity except with my expressed written consent.

MY CONSENT IS GIVEN VOLUNTARILY. THEREFORE, MY SIGNATURE INDICATES THAT I HAVE DECIDED TO PARTICIPATE IN THIS STUDY UNDERSTANDING ALL THE INFORMATION PROVIDED TO ME IN THIS FORM AND THAT I HAVE RECEIVED A COPY OF THIS CONSENT FORM.

Participant Signature Date

Print your name

Investigator Signature

Witness Signature 
82

APPENDIX C

TRAINING PROTOCOL WITH ONE MINUTE REST INTERVALS 


\begin{tabular}{|c|c|c|}
\hline \multicolumn{3}{|c|}{ Appendix } \\
\hline Training Pro & ol \# 1 (TP1) & \\
\hline Prescription: & 10RM & \\
\hline Sets: & 3 & \\
\hline Rest Periods: & 1 minutes between & sets and exercises (* \\
\hline Exercises: & 1. Bench Press & $10 * 10 * 10 *$ \\
\hline & 2. Leg Press & $10 * 10 * 10 *$ \\
\hline & 3. Lat Pull-down & $10 * 10 * 10 *$ \\
\hline & 4. Leg Curl & $10 * 10 * 10 *$ \\
\hline & 5. Military Press & $10 * 10 * 10 *$ \\
\hline & 6. Upright Rowing & $10 * 10 * 10 *$ \\
\hline & 7. Leg Extension & $10 * 10 * 10 *$ \\
\hline & 8. Tricep Pushdown & $10 * 10 * 10 *$ \\
\hline & 9. Calf Raises & $10 * 10 * 10 *$ \\
\hline & 10. Arm Curl & $10 * 10 * 10 *$ \\
\hline
\end{tabular}


APPENDIX D

TRAINING PROTOCOL WITH THREE MINUTE REST INTERVALS 


\section{Appendix D}

Training Protocol \# 3 (TP3)

Prescription:

Sets: $\quad 3$

Rest Periods: 3 minutes between sets and exercises

Exercises: 1. Bench Press $10 * 10 * 10 *$

\begin{tabular}{|c|c|}
\hline 2. Leg Press & $10 * 10 * 10 *$ \\
\hline 3. Lat Pull-down & $10 * 10 * 10$ \\
\hline 4. Leg Curl & $10 * 10 * 10$ \\
\hline 5. Military Press & $10 * 10 * 10$ \\
\hline
\end{tabular}

6. Upright Rowing $10 * 10 * 10 *$

7. Leg Extension $10 * 10 * 10 *$

8. Tricep Pushdown $10 * 10 * 10 *$

9. Calf Raises $\quad 10 * 10 * 10 *$

10. Arm Curl $10 * 10 * 10 *$ 


\section{APPENDIX E}

STANDARDIZED WARM-UP PROTOCOL 


\section{Appendix E}

\section{Standardized Warm-up Protocol}

The following preliminary exercises will be performed:

Stretching (statically) exercises for flexibility

-standing floor touch

-sitting toe touch

-alternate toe touch

-lower-leg stretch

-upper chest stretch

-upper arm stretch

\section{Calisthenics}

-jumping jacks

-toe raises

-leg raises

-half squats

-push-ups

-bent-knee sit-ups

Formal activity

-Mimic same exercises as in the HRE protocol with low intensity and low volume ( $40 \%$ of $1 \mathrm{RM}, 4-5$ repetitions).

Note: the total warm-up time will be of approximately 15 minutes 
APPENDIX F

STANDARDIZED COOL-DOWN PROTOCOL 


\section{Appendix $\mathrm{F}$}

\section{Standardized Cool-down Protocol}

The following exercises will be performed:

Formal activity

-Mimic same exercises as in the HRE protocol with low intensity and low volume ( $40 \%$ of $1 \mathrm{RM}, 3-4$ repetitions).

\section{Calisthenics}

-jumping jacks

-toe raises

-leg raises

Stretching (statically) exercises for flexibility

-standing floor touch

-sitting toe touch

-alternate toe touch

-lower-leg stretch

-upper chest stretch

-upper arm stretch

Note: The formal activity and the stretching exercises will have a major emphasis than calisthenics. 
Appendix G

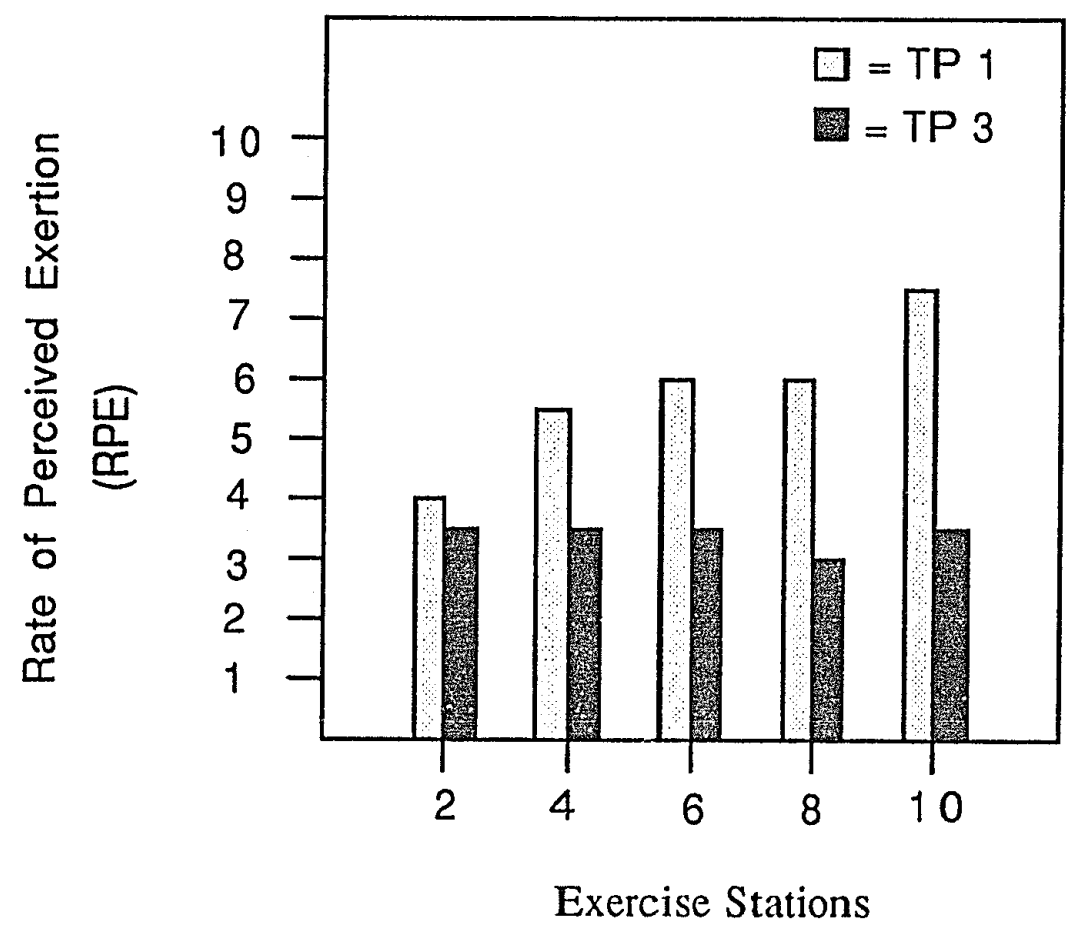

Fig 1 Comparison of ratings of perceived exertion of the subjects to the one (TP1) and three (TP3) min rest interval workouts. 
APPENDIX $\mathrm{H}$

HEART RATE RESPONSES FIGURE 
Appendix $\mathrm{H}$

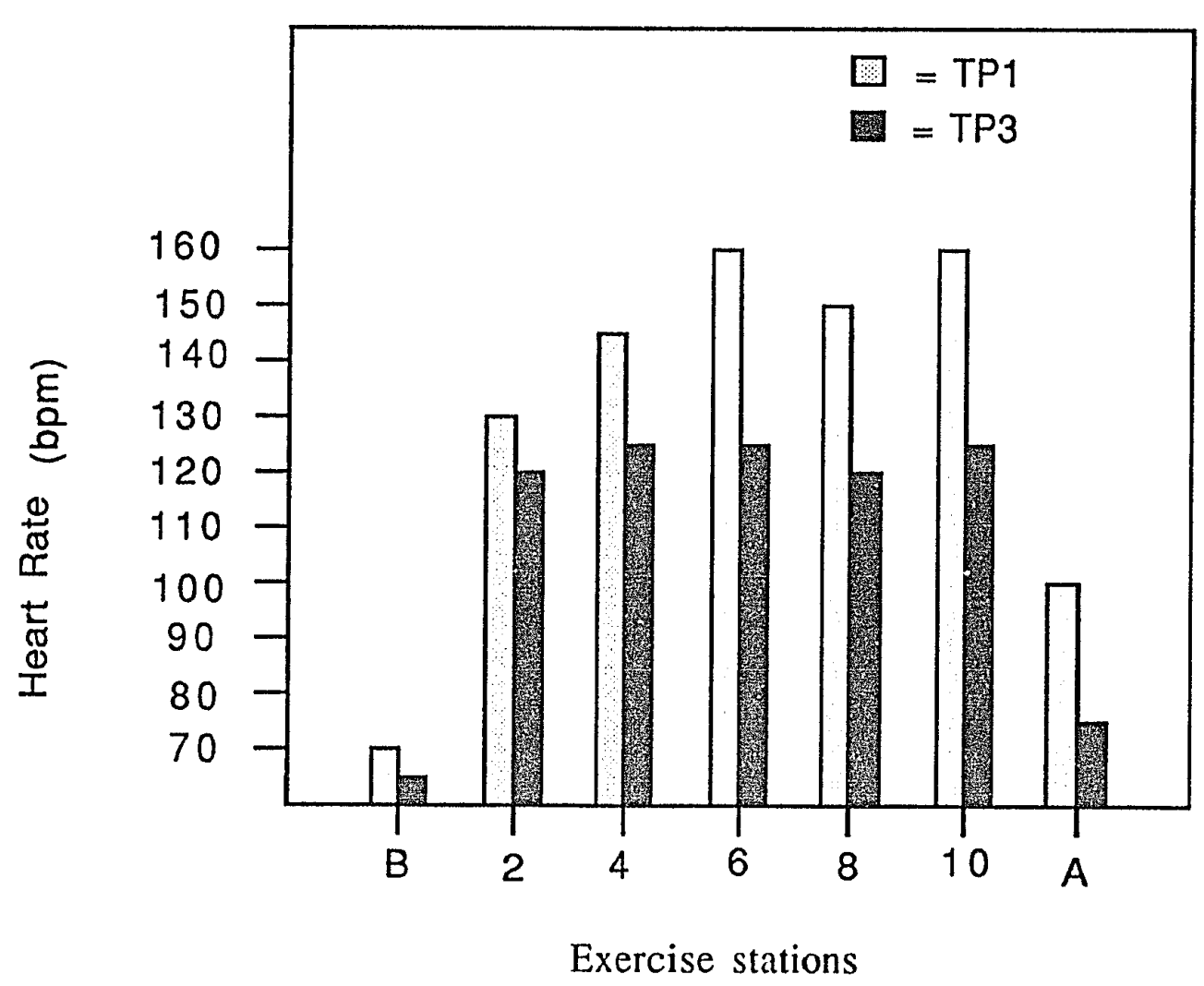

Fig 2 Comparision of heart rate responses before (B), during the exercise stations with one (TP1) and three (TP3) min rest intervals, and after (A) exercise. 
APPENDIX I

BLOOD LACTATE CONCENTRATION LEVELS 


\section{APPENDIX I}

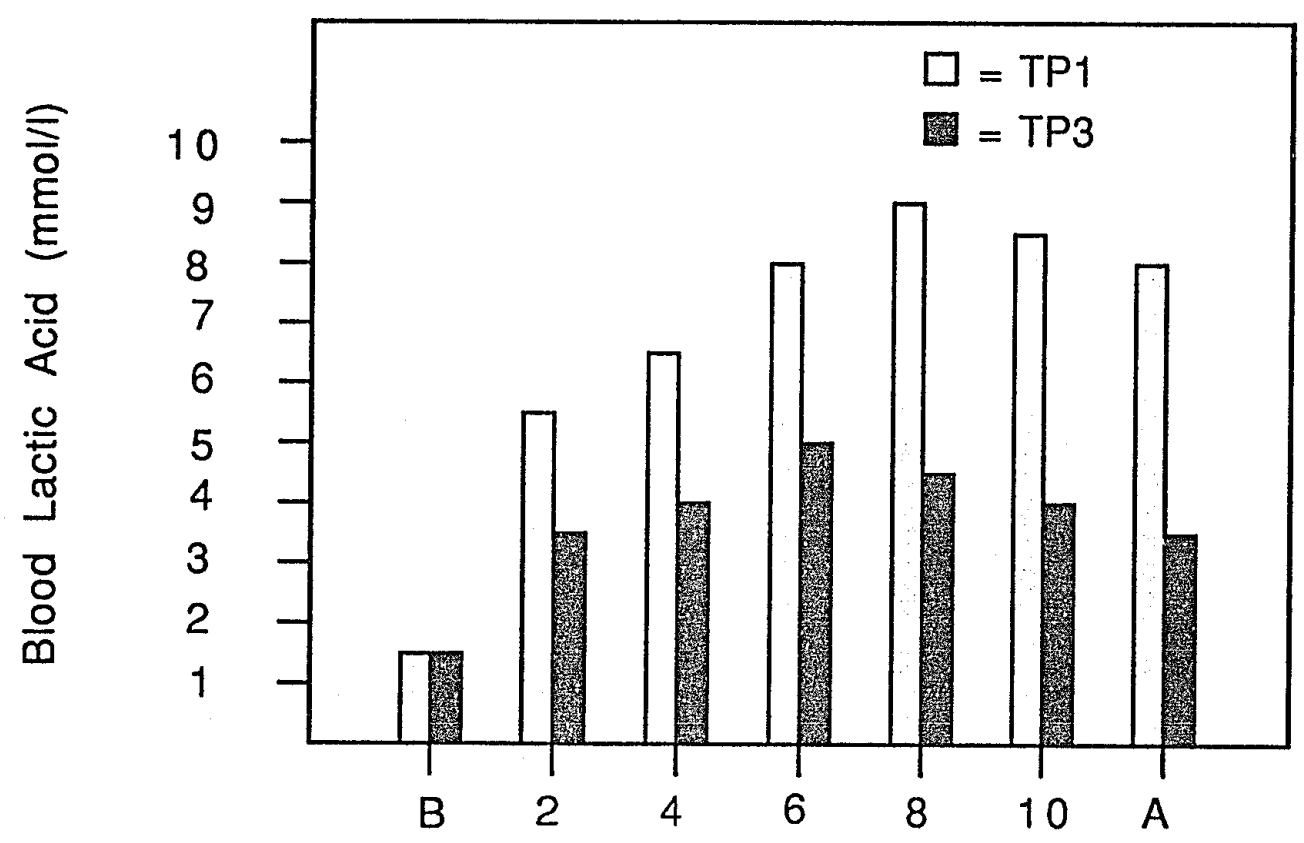

\section{Exercise Stations}

Fig 3 Comparison of blood lactate concentration (bla) before (B), during the exercise stations with one (TP1) and three (TP3) min rest interval, and after (A) exercise. 
APPENDIX J

SYSTOLIC BLOOD PRESSURE RESPONSES 
APPENDIX $\mathbf{J}$

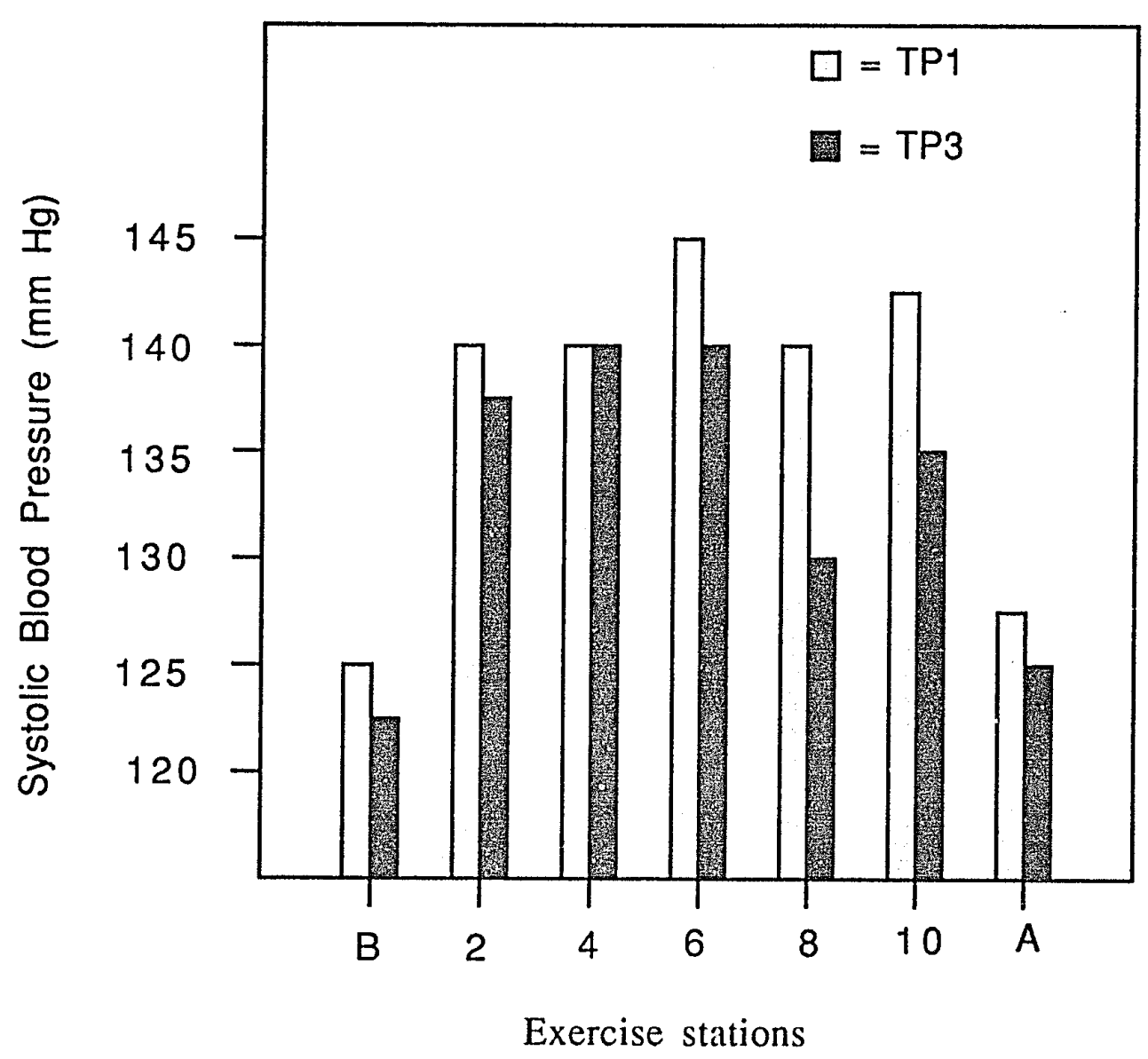

Fig 4 Comparison of systolic blood pressure before (B), during the exercise stations with one (TP1) and three (TP3) min rest interval, and after (A) exercise. 
APPENDIX K

CARDIAC COST RESPONSES 
APPENDIX K

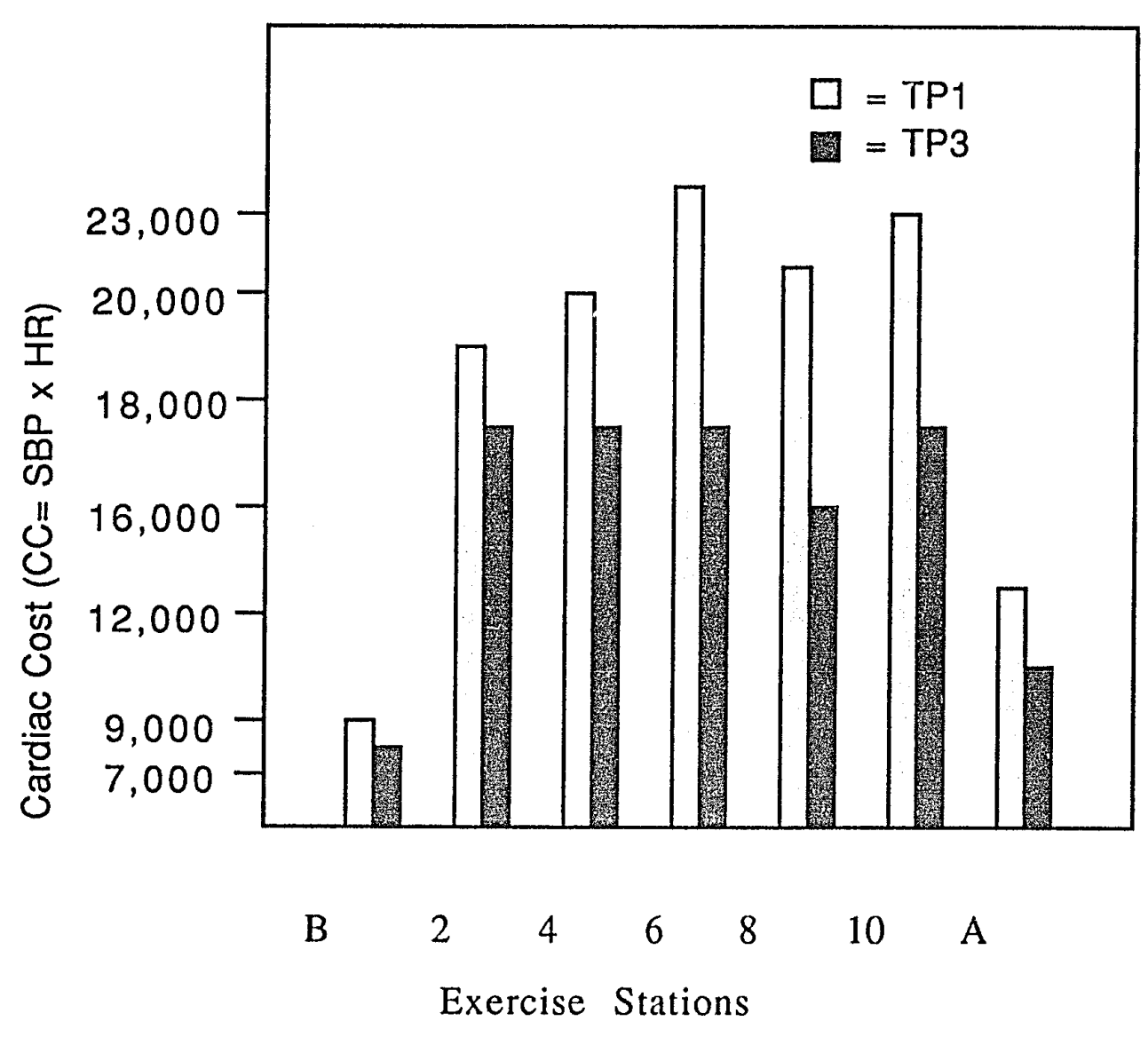

Fig 5 Comparison of cardiac cost before (B), during the exercise stations with one (TP1) and three (TP3) min rest interval, and after (A) exercise. 
APPENDIX L

\section{ANALYSIS OF VARIANCE SUMMARY TABLES}




\begin{abstract}
APPENDIX L-1. ANOVA Summary Table for Blood Lactate Concentration During Training Protocols.
\end{abstract}

\begin{tabular}{|c|c|c|c|c|c|}
\hline SOURCE & SS & DF & MS & $\mathrm{F}$ & SIGNIFICANCE \\
\hline Within Cells & 25.74 & 10 & 2.57 & & \\
\hline Protocols & 314.86 & 1 & 314.86 & 122.32 & .001 \\
\hline TOTAL & 340.60 & 11 & & & \\
\hline SOURCE & SS & $\mathrm{DF}$ & MS & $\mathrm{F}$ & SIGNIFICANCE \\
\hline Within Cells & 75.59 & 60 & 1.26 & & \\
\hline Workouts & 447.01 & 6 & 74.50 & 59.14 & .001 \\
\hline TOTAL & 522.6 & 66 & & & \\
\hline SOURCE & SS & $\mathrm{DF}$ & MS & $\mathrm{F}$ & SIGNIFICANCE \\
\hline Within Cells & 33.81 & 60 & .56 & & \\
\hline Interaction & 93.17 & 6 & 15.53 & 27.55 & .001 \\
\hline
\end{tabular}




\section{APPENDIX L-2. MANOVA Summary Table for Heart Rate Responses During Training Protocols}

$\begin{array}{lllllc}\text { SOURCE } & \text { SS } & \text { DF } & \text { MS } & \text { F } & \text { SIGNIFICANCE } \\ \text { Within Cells } & 1154.80 & 9 & 128.31 & & \\ \text { Regression } & 121.46 & 1 & 121.46 & .95 & .356 \\ \text { Protocols } & 10758.30 & 1 & 10758.30 & 83.85 & .001 \\ & & & & & \end{array}$

\begin{tabular}{|c|c|c|c|c|c|}
\hline SOURCE & SS & $\mathrm{DF}$ & MS & $\mathrm{F}$ & SIGNIFICANCE \\
\hline Within Cells & 6135.83 & 50 & 122.72 & & \\
\hline Workouts & 43788.10 & 5 & 8757.62 & 71.36 & .001 \\
\hline
\end{tabular}

$\begin{array}{llllll}\text { SOURCE } & \text { SS } & \text { DF } & \text { MS } & \text { F } & \text { SIGNIFICANCE } \\ \text { Within } & 3512.59 & 50 & 70.25 & & \\ \text { Interaction } & 2258.64 & 5 & 451.73 & 6.43 & .001 \\ \ldots & & & & \end{array}$


APPENDIX L-3. ANOVA Summary Table for the Rating of Perceived Exertion During Training Protocols

\begin{tabular}{|c|c|c|c|c|c|}
\hline SOURCE & SS & DF & MS & $\mathrm{F}$ & SIGNIFICANCE \\
\hline Within Cells & 59.80 & 10 & 5.98 & & \\
\hline Protocols & 133.10 & 1 & 133.10 & 22.26 & .001 \\
\hline
\end{tabular}

\begin{tabular}{|c|c|c|c|c|c|}
\hline SOURCE & SS & DF & MS & $\mathrm{F}$ & SIGNIFICANCE \\
\hline Within Cells & 52.22 & 40 & 1.31 & & \\
\hline Workouts & 45.58 & 4 & 11.40 & 8.73 & .001 \\
\hline
\end{tabular}

$\begin{array}{lllllc}\text { SOURCE } & \text { SS } & \text { DF } & \text { MS } & \text { F } & \text { SIGNIFICANCE } \\ \text { Within Cells } & 45.02 & 40 & 1.13 & & \\ \text { Interaction } & 31.58 & 4 & 7.90 & 7.02 & .001 \\ & & & & & \\ \text { TOTAL } & 76.60 & 44 & & & \end{array}$


APPENDIX L-4. ANOVA Summary Table for Systolic Blood Pressure Responses During Training Protocols

$\begin{array}{llllll}\text { SOURCE } & \text { SS } & \text { DF } & \text { MS } & F & \text { SIGNIFICANCE }\end{array}$

Within Cells $\quad 3167.57 \quad 10 \quad 316.76$

$\begin{array}{llllll}\text { Protocols } & 804.57 & 1 & 804.57 & 2.54 & .142\end{array}$

$\begin{array}{lll}\text { TOTAL } & 3972.14 \quad 11\end{array}$

$\begin{array}{llllll}\text { SOURCE } & \text { SS } & \text { DF } & \text { MS } & F & \text { SIGNIFICANCE }\end{array}$

Within Cells $\quad 3384.06 \quad 60 \quad 56.40$

$\begin{array}{llllll}\text { Workouts } & 7296.22 & 6 & 1216.04 & 21.56 & .001\end{array}$

$\begin{array}{lll}\text { TOTAL } & 10680.28 & 66\end{array}$

$\begin{array}{llllll}\text { SOURCE } & \text { SS } & \text { DF } & \text { MS } & F & \text { SIGNIFICANCE }\end{array}$

Within Cells $2096.7960 \quad 34.95$

$\begin{array}{llllll}\text { Interaction } & 392.06 & 6 & 65.34 & 1.87 & .101\end{array}$

TOTAL

$2488.85 \quad 66$ 
APPENDIX L-5. ANOVA Summary Table for Cardiac Cost Responses During Training Protocols

$\begin{array}{llllll}\text { SOURCE } & \text { SS } & \text { DF } & \text { MS } & \text { F } & \text { SIGNIFICANCE } \\ \text { Within Cells } & 51920020.17 & 9 & 5768891.1 & & \\ \text { Regression } & 32462121.36 & 1 & 32462121 & 5.63 & .042 \\ \text { Protocols } & 174082802.1 & 1 & 174082802 & 30.18 & .001 \\ & & & & & \end{array}$

$\begin{array}{llllll}\text { SOURCE } & \text { SS } & \text { DF } & \text { MS } & \text { F } & \text { SIGNIFICANCE } \\ \text { Within Cells } & 144435799.5 & 50 & 2888716.0 & & \\ \text { Workouts } & 1213775170 & 5 & 242755034 & 84.04 & .001\end{array}$

TOTAL $\quad 1616675913 \quad 55$

\begin{tabular}{|c|c|c|c|c|c|}
\hline SOURCE & SS & DF & MS & $\mathrm{F}$ & SIGNIFICANCE \\
\hline Within Cells & 115872657.8 & 50 & 2317453.2 & & \\
\hline Interaction & 75820969.83 & 5 & 15164194 & 6.54 & .001 \\
\hline
\end{tabular}

